\title{
Role of Damage-Associated Molecular Patterns in Light of Modern Environmental Research: A Tautological Approach
}

\author{
Walter Gottlieb Land ${ }^{1,2}$ (D)
}

Received: 13 May 2020 / Revised: 30 July 2020 / Accepted: 1 August 2020 / Published online: 9 August 2020

(c) University of Tehran 2020

\begin{abstract}
Two prominent models emerged as a result of intense interdisciplinary discussions on the environmental health paradigm, called the "exposome" concept and the "adverse outcome pathway" (AOP) concept that links a molecular initiating event to the adverse outcome via key events. Here, evidence is discussed, suggesting that environmental stress/injury-induced damageassociated molecular patterns (DAMPs) may operate as an essential integrating element of both environmental health research paradigms. DAMP-promoted controlled/uncontrolled innate/adaptive immune responses reflect the key events of the AOP concept. The whole process starting from exposure to a distinct environmental stress/injury — associated with the presence/ emission of DAMPs - up to the manifestation of a disease may be regarded as an exposome. Clinical examples of such a scenario are briefly sketched, in particular, a model in relation to the emerging COVID-19 pandemic, where the interaction of noninfectious environmental factors (e.g., particulate matter) and infectious factors (SARS CoV-2) may promote SARS case fatality via superimposition of both exogenous and endogenous DAMPs.
\end{abstract}

\section{Article Highlights}

- This review is devoted to the first scientific attempt to integrate DAMP-controlled innate immune inflammatory and adaptive immune defense responses as well as DAMP-uncontrolled dysregulated pathological responses (acute and chronic human diseases) in the environmental health paradigm, called the "exposome" concept and the "adverse outcome pathway" (AOP) concept.

- Evidence is discussed that DAMP-promoted controlled/uncontrolled innate immune/adaptive immune responses reflect the key events of the AOP concept.

- The whole process starting from exposure to a distinct environmental stress/injury—associated with the presence/ emission of DAMPs - up to the manifestation of a disease may be regarded as an exposome.

- Some exemplifying models of such a scenario are briefly sketched, including respiratory infections, autoimmune/ allergic diseases, organ fibrosis, and cancer.

- In particular, a model in relation to the emerging COVID-19 pandemic is presented, where the interaction of noninfectious environmental factors (e.g., particulate matter) and infectious factors (SARS CoV-2) may promote SARS case fatality via superimposition of both exogenous and endogenous DAMPs.

Keywords DAMPs $\cdot$ Innate immunity $\cdot$ Exposome $\cdot$ Adverse outcome pathway $\cdot$ COVID-19

Walter Gottlieb Land

Gottliebland@gmail.com

https://www.akademie-fuer-transplantationsmedizin.de/

1 German Academy for Transplantation Medicine, Munich, Germany

2 Molecular ImmunoRheumatology, Laboratory of Excellence Transplantex, Faculty of Medicine, INSERM UMR_S1109, University of Strasbourg, Strasbourg, France

\section{Introduction}

The most common diseases result from the combined effect of genes and environmental factors and the interactions between them. The study of the whole genome (genomics) has tremendously evolved into a multidisciplinary area of science with highly diverse applications in medical genetics (National Human Genome 2018). Considerable improvement 
emerged for genome sequencing since the next-generation sequencing (NGS) approaches developed in the 2000s, thereby allowing genomic assessments in large study populations (Johansen Taber et al. 2014; Goldfeder et al. 2017. The newer technologies enable large-scale whole-genome sequencing (WGS) accessible and practical for researchers. Today, WGS is increasingly prevalent in detecting the genetics of human diseases, thereby guiding disease prevention, clinical diagnoses, and therapeutic intervention (Green and Guyer 2011). Thousands of genome-wide association studies (GWAS) have tracked relationships between base pair/gene patterns in genomic loci and hundreds of human diseases (National Human Genome 2018; Goodwin et al. 2016).

However, although enormous costs have been dedicated to discovering relevant disease-related genetic variants, especially in GWASs, only a small fraction of estimated heritability can be explained by these results, called the missing heritability problem (Otani et al. 2018). Also, it has become apparent that genes alone account for the minority of disease etiology for many critical disorders and that most chronic diseases are attributable to both genetic and environmental influences (Theodoratou et al. 2017). Even more striking, the environment has now been recognized to be the major contributor to the global disease burden (Rappaport and Smith 2010; Manrai et al. 2017). Notably, 80-85\% of human diseases are reportedly linked to environmental exposures (Uppal et al. 2016). Even the current outbreak of novel coronavirus COVID-19 (HCoV-19 or SARS-CoV-2) has been linked to environmental factors ( $\mathrm{Qu}$ et al. 2020). The authors claim: "In addition to expertise in the fields of medicine, public health, and computer science, the contribution of environmental scientists in collaborative research is urgently warranted for combating the infectious disease threat at a global scale." However, quantifying and specifying the myriad of environmental and lifestyle risk factors such as smoking, exposure to hazardous chemicals, and pathogenic microorganisms turned out to be very complex and challenging to manage (Rappaport and Smith 2010; Willett 2002). For example, persons can be exposed to a complex mixture of chemical and biological contaminants, with multiple sources, for varying durations across their life course.

As a result of finding a way out of this dilemma, the continuous endeavor of environmental research was intensified and culminated in the development of the "environmental health paradigm" (Wilson and Schwartz 2006). This approach tries to integrate the knowledge of exposures and environmental health sciences by exploring the causal links between environmental exposure and adverse disease effects. Two prominent models emerged as a result of intense interdisciplinary discussions on the environmental health paradigm, called the "exposome" concept and the "adverse outcome pathway" (AOP) concept. Under the exposome paradigm, all non-genetic factors contributing to disease are considered to be "environmental factors". Of note, during the last decade, the exposome approach has mainly been considered in epidemiology, while the other complementary concept of AOP has predominantly emerged in (eco) toxicology. The concept of the environmental health paradigm - academically covered by the new field of exposure science (Lioy and Smith 2013) - has worldwide intensified efforts of modern environmental research to study adverse effects of environmental agents on humans and animals. The main subject of such cross-disciplinary studies is the search for new tools to assess the risk of adverse health outcomes caused by harmful environmental factors. Indeed, the field of environmental research is flourishing, as reflected by the establishment of an interdisciplinary area of environmental health sciences and public health.

Excitingly, there is accumulating evidence in support of the notion that damage-associated molecular patterns (DAMPs) - defined as any molecule sensed or recognized by a cognate pattern recognition molecule (PRM) on/in cells of the innate immune defense system-are an essential integrating element of both concepts. This recognition appears to be reason enough to address the three models in a special review article, here, in light of the danger/injury model in Immunology (Matzinger 1994, 2012; Land et al. 1994; Land 2003, 2018; Seong and Matzinger 2004; Land and Messmer 2012, 2013).

\section{DAMPs in Host Defense and Diseases at a Glance}

\section{DAMPs: The Protagonists of Host Innate Immune Defense}

DAMPs are molecules that are generated and emitted upon any cell stress and/or tissue injury, even the slightest intraand extracellular molecular perturbations. The DAMPs, a term introduced in 2003/2004 (Land 2003; Seong and Matzinger 2004), can be regarded as the core of the danger/injury theory in Immunology proposed in 1994 (Matzinger 1994; Land et al. 1994). In perspective from and addressed in our book "DAMPs in Human Diseases" (see Land (2018), Chpt. 2 , p.13), the model claims that any form of cell stress and tissue injury, including pathogen-mediated infectious stress/ injury,- - via generation and emission of DAMPs—elicit a robust host defense response. DAMPs have gained particular attention in the context of our increasing understanding that dying cells actively regulate not only inflammatory but also adaptive immune responses (Yatim et al. 2017; Nagata and Tanaka 2017). Moreover, current notions even hold that all organisms on our planet use DAMPs for their defense against any cell stress/tissue injury (Heil and Land 2014). 
Hence, the evolutionarily determined function of DAMPs is to promote injury-induced defense responses, the aim always being to eliminate the injurious agent and repair damaged tissue, that is, to restore homeostasis: "DAMPs across the tree of life".

In mammals, four categories of DAMPs can be defined, termed as (I) endogenous constitutively expressed native DAMPs such as high mobility group box 1 (HMGB1), S100 proteins, cell-free endogenous nucleic acids (NAs), and histones; (II) endogenous constitutively expressed, injurymodified DAMPs, including cell-extrinsic modified DAMPs such as oxidation-specific epitopes (OSEs), and cell-intrinsic modified DAMPs such as dyshomeostasis-associated molecular patterns [also called homeostatic danger signals (Gallo and Gallucci 2013)]; (III) endogenous inducible DAMPs produced by previously DAMP-activated stressed or dying cells such as interleukin-1beta (IL-1 $\beta$ ), tumor necrosis factor (TNF), and type I interferons (IFNs); and (IV) exogenous DAMPs such as airborne particulate matter (PM) [for details of their definitions and functions, see Land (2018), Chpts. 11-16, pp. 191-370].

The DAMPs accomplish their function in promoting injury-induced defense responses by activating various PRM-bearing mobile and sessile cells of the innate immune system, including leukocytes, macrophages, dendritic cells (DCs), innate lymphoid cells, epithelial and endothelial cells, and fibroblasts. Together with soluble humoral recognition receptors such as complement fragment $\mathrm{C} 1 \mathrm{q}$ and pentraxins, all these cellular PRMs impressively identify the innate immune defense system as a highly efficient organ of perception [described in detail in Land (2018), Chpt. 4, p. 33 and Chpt. 5, p. 43].

The work of DAMPs is complex and consists of inflammation-promoting and inflammation-resolving capabilities, the aim always is to restore and maintain homeostasis upon any injury. In brief: initial cell stress/tissue damage induces molecular perturbation reflecting the first line of DAMPs (usually dyshomeostatic DAMPs), which elicit cell-autonomous stress responses such as autophagy, oxidative stress response, heat shock response (HSR), DNA damage response (DDR), and the endoplasmic reticulum (ER)-induced unfolded protein response (UPR) [described in Land (2018). Chpt. 18, p. 377]. When these responses fail to restore cellular homeostasis, they can result in the development of subroutines of regulated cell death (RCD) that are associated with passive release of large amounts of constitutive DAMPs (Land et al. 2016; Sarhan et al. 2018) [for details of RCD, also see Land (2018), Chp. 19, p. 427]. These DAMPs - via activation of PRM-bearing cells-promote context-dependently proinflammatory and-in their function as suppressing DAMPs (SAMPs)—inflammationresolving responses; additionally, they drive profibrotic tissue-repairing processes. Also, in order to enrich the defensively oriented arsenal of DAMPs, DAMP-activated PRM-expressing cells (or cells succumbing from RCD) secret cytokines such as TNF and type I IFNs, which may act as inducible DAMPs (for TNF and type I IFNs denoted as inducible DAMPs, see Land (2018), Sect. 14.3.2, p. 322, and Sect. 14.2.3, p. 312]. Moreover, in the presence of alteredself or nonself antigens, the DAMPs - via activation of antigen-presenting DCs-shape adaptive immune responses, which have the same homeostasis-restoring aim [for details and more information on DAMP-promoted innate and adaptive immune responses as well as profibrotic/repairing processes, see Land (2018), Chpts. 21-25, pp. 473-659, Chpts. 30-32, pp. 717-790, and Chpt. 36, p. 845].

\section{The Dark Side of DAMPs: Promotion of Pathologies and Inflammatory Diseases}

The powerful defense responses elicited by DAMPs is good news; however, there is also bad news. Under uncontrolled and dysregulated conditions, the DAMP-triggered, PRM-mediated responses can result in pathologies such as the development of chronic inflammatory, autoimmune, or neurodegenerative diseases. But even worse: when DAMPs are emitted uncontrolled in excess and released locally and/or systemically in large amounts, for example, in severe local or systemic tissue injury, an acute exaggerated, local and/or systemic hyperinflammatory response may develop. Mechanistically, the involvement of five lines of DAMPs can be sketched and discussed in this scenario (Fig. 1) [reviewed in Land (2018), Chpt. 20. p. 467]: Initial tissue injury, via generation of the first line of dyshomeostatic DAMPs, elicits cell-intrinsic stress responses that, when unsuccessful in restoring homeostasis, result in RCD (associated with release of a third line of constitutive DAMPs), the emission of a second line of DAMPs serving as the medium. Such a second line of DAMPs, for example, refers to thioredoxin-interacting protein (TXNIP) that is rapidly generated in the ER stress $\rightarrow$ UPR pathway and represents a critical node in the terminal UPR. In turn, it can activate the NLRP3 inflammasome resulting in pyroptosis (Lerner et al. 2012; Sano and Reed 2013; Heo et al. 2019) (NLRP3 stands for nucleotide-binding and oligomerization domain-like receptor [NLR] family pyrin domain-containing 3). There is another second line of DAMPs that can also be discussed: During ER stress, calcium homeostasis in the ER is imbalanced, and $\mathrm{Ca}^{2+}$ is released from the ER into the cytoplasm via several channels, in particular ryanodine receptors and inositol 1,4,5-trisphosphate receptors (Sharp et al. 1993). Subsequently, increased calcium concentration in the cytoplasm then has been shown to activate the NLRP3 inflammasome [reviewed in Chen et al. (2019a) and Swanson et al. (2019)]. The disturbed $\mathrm{Ca}^{2+}$-mediated intracellular 


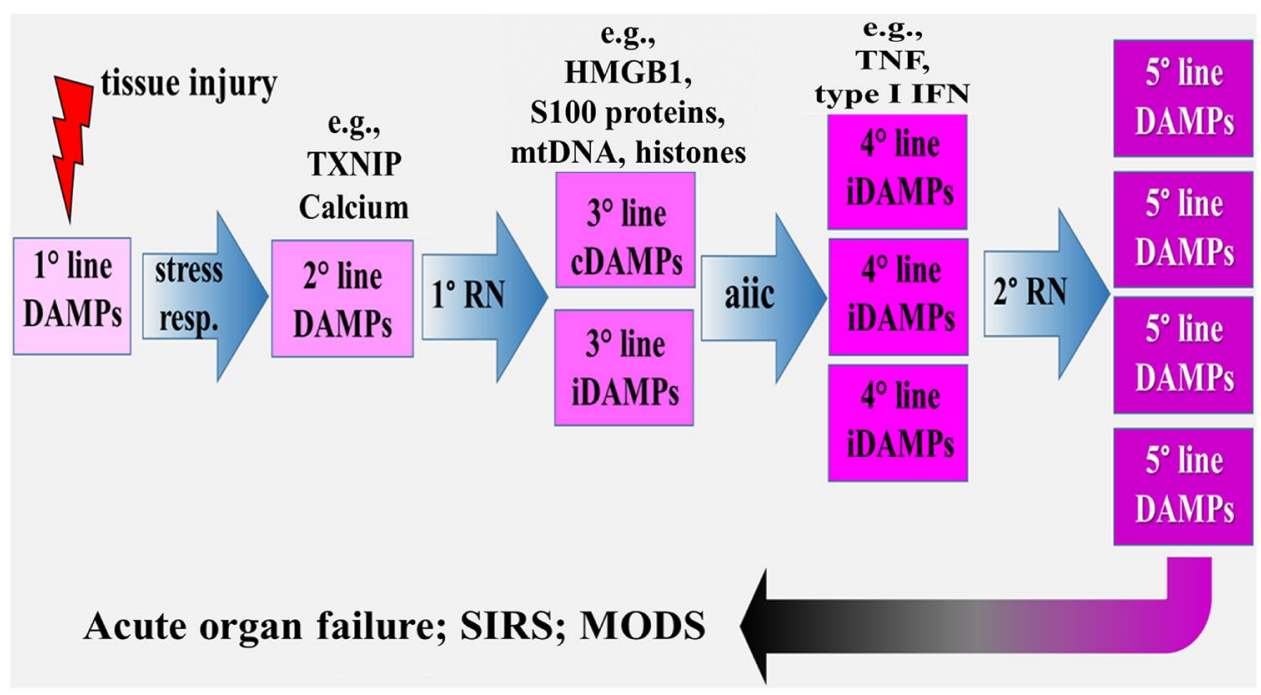

Fig. 1 Conceptual scenario model of excessive DAMPs emission that may avalanche-like develop into a chain of five lines of different DAMPs, promoting and expanding acute local/systemic hyperinflammatory responses. Clinically, these processes manifest as acute solid organ failure and/or systemic inflammatory response syndrome, which may lead to multiple organ dysfunction syndrome. aiic activated innate immune cells, $c D A M P s$ constitutive DAMPs, $H M G B 1$

molecular perturbation again reflects dyshomeostatic DAMPs, which are also sensed by NLRP3 to activate the inflammasome. The fourth line of DAMPs refers to inducible DAMPs such as TNF, which are secreted by DAMPactivated or dying cells. Both constitutive and inducible DAMPs can overlap and result in an overshooting, hyperinflammatory process that may drive a secondary round of RCD associated with the release of a fifth line of DAMPs, a phenomenon called "propagation of RCD" (Kaczmarek et al. 2013; Newton 2015) [for more information about RCD, see Sarhan et al. (2018), Land (2018), Chpts. 18-20, pp. 377-470].

Clinically, local and/or systemic DAMP-promoted hyperinflammatory responses are diagnosed as acute solid organ failure, for example, as acute lung injury (ALI) and acute respiratory distress syndrome (ARDS) [reviewed, e.g., in Tolle and Standiford (2013) and Englert et al. (2019)], or as sepsis in terms of a systemic inflammatory response syndrome (SIRS) that can lead to multiple organ dysfunction syndrome (MODS), often associated with patient's death [reviewed, e.g., in Relja and Land (2019), Eppensteiner et al. (2019)]. Importantly, there is accumulating evidence indicating that DAMPs such as HMGB1, cell-free DNA, and histones contribute to the development of sepsis-associated MODS not only via promotion of hyperinflammatory but also procoagulant pathways. Therefore, these molecules are also essentially involved high mobility group box 1 , iDAMPs inducible DAMPs, IFN interferon, MODS multiple organ dysfunction syndrome, mtDNA mitochondrial DNA, $R N$ regulated necrosis, stress resp stress responses, SIRS systemic inflammatory response syndrome, $T N F$ tumor necrosis factor, TXNIP thioredoxin-interacting protein. Source: The figure was published in Land (2018)

in the pathogenesis of disseminated intravascular coagulation (DIC) (Liaw et al. 2016; Toh et al. 2016).

\section{The Exposome Concept}

\section{Introductory Remarks}

The introduction of the term "exposome" is an emerging attempt to conceptualize and characterize the whole spectrum of exposures humans encounter from birth to death. Accordingly, the term "exposome" describes the totality of environmental exposures to which an individual is subjected from conception to death. It includes both external and internal factors, as well as the human body's response to these factors (Rappaport and Smith 2010; Wild 2012). Hence, under the exposome paradigm, all non-genetic factors contributing to disease are considered to be "environmental" including chemicals, drugs, infectious agents such as viruses, and psychosocial stress [for reviews and competent articles on the exposome, see Rappaport and Smith (2010), Wild (2012, 2005), Rappaport (2011), National Research Council (NRC) (2011), Miller and Jones (2014), Smith et al. (2015), Siroux et al. (2016), Johnson et al. (2017), Patel (2017), Rattray et al. (2018), US (2019), Escher et al. (2017), Vineis et al. (2020), Vermeulen et al. (2020) and Santos et al. (2020)]. 
Historically, the term "exposome" was coined by Wild (2005) to describe the totality of environmental exposures to which an individual is subjected from conception throughout the life course to final death. The author understood the exposome as a complement to the genome that may be helpful in understanding environmental factors to contribute to the etiopathogenesis of chronic diseases. Wild (2005) concluded, “... The concept of an exposome may serve to highlight this requirement and to balance the effort going towards characterization of the genome. An extension of the current generation of biomarkers, together with an evaluation of the new generation of "omics" technologies, has a crucial role to play in this regard .... However, advances will require increasing collaboration between epidemiologists, biostatisticians, experts in bioinformatics, and laboratory and environmental scientists. In addition, funding agencies must take a medium- to long-term view and encourage research that focuses on improved measures of environmental risk factors, an area that currently seems to be less of a priority for support than many others in the broad domain of medical research."

The approach of Wild was modified by Rappaport and Smith (2010) and Rappaport (2011), who stressed that exposures are not only comprised of toxic chemicals entering the body from the environment, such as pollutants, reactive electrophiles, and metal. In fact, the authors extended the term exposome by including human exposures from all exogenous and endogenous sources in the body's "internal chemical environment". In their 2011 paper (Rappaport 2011), they wrote: "Thus, it would be reasonable to consider the "environment" as the body's internal chemical environment and "exposures" as the amounts of biologically active chemicals in this internal environment. Under this view, exposures are not restricted to chemicals (toxicants) entering the body from air, water, or food, for example, but also include chemicals produced by inflammation, oxidative stress, lipid peroxidation, infections, gut flora, and other natural processes... This internal chemical environment continually fluctuates during life due to changes in external and internal sources, aging, infections, life-style, stress, psychosocial factors, and preexisting diseases".

In the meantime, the exposome has moved from a concept to a reality and was recently redefined as "the cumulative measure of environmental influences and associated biological responses throughout the lifespan, including exposures from the environment, behavior, diet, and endogenous processes" (Miller and Jones 2014).

Today, the progress in the characterization of the exposome allows a better understanding of the complex interplay of environmental factors with genetic susceptibility. Indeed, the gained knowledge is helpful in elucidating the epidemiological phenomenon that the majority of genetic alterations will contribute to population disease burden only in the presence of specific environmental exposures.

\section{The Exposome and Exposomics}

Research on the exposome has been further refined by introducing the new field of "exposomics" that is understood as the comprehensive analysis of exposure to all environmental chemical and non-chemical stress factors (=stressors). As outlined by Smith et al. (2015), the goal of exposomics research is to measure, in human bodily fluids, all "small signaling molecules" (in terms of biologically active chemicals) which are involved in mediating the effects of stressors on the body by altering cellular activity and physiological processes. Further, exposomics science should attempt to link the presence of these small molecules with functional changes in biology, leading to chronic illnesses. The authors (Smith et al. 2015) discuss further:... "The internal measurements made in exposomics could be of individual chemicals, groups of chemicals or the totality of chemicals acting on a particular receptor or biological pathway in a functional assay. Hence, exposomics can be operationalized by studying all the small molecules in the body and their influence on biological pathways that lead to impaired health." The authors continue with an epidemiological discourse by arguing that the health of a given community, and the individuals within it, is dependent on a variety of environmental and social factors (Smith et al. 2015). In this context, the issue of cumulative risk assessment is addressed as defined by the United States Environmental Protection Agency (US EPA) (US 2019): "Combined risks from aggregate exposures to multiple agents or stressors, where agents or stressors may include chemical and nonchemical stressors." Indeed, this statement refers essentially to the exposome paradigm where all non-genetic environmental stressors are considered. Hence, cumulative risk assessment, where the impact of all stressors on a population is assessed, appears to be a burning topic to be standardized and specified by exposomics. In other words: The exposome has been advocated as a key to cumulative risk assessment (Smith et al. 2015). Recently, the concept has been redefined by Rappaport (2018): "The blood exposome consists of chemicals derived from both endogenous and exogenous sources. Endogenous chemicals are represented by the human proteome and metabolome, which establish homeostatic networks of functional molecules. Exogenous chemicals arise from diet, vitamins, drugs, pathogens, microbiota, pollution, and lifestyle factors, and can be measured in blood as subsets of the proteome, metabolome, metals, macromolecular adducts, and foreign DNA and RNA." According to this definition, the 
exposome is specific for individuals and integrates lifetime exposure while the AOP is conceptually focused on biological mechanisms and pathways-as will be further alluded to in the following sections.

\section{Some Examples of Exposomes as Reported in the Literature}

\section{General Remarks}

Though research on exposomes is probably still in its infancy, some reports have already been published regarding the description of some subroutines. Indeed, mixtures of various exposures, or combinations and interactions between multiple environmental exposures, are supposed to be causally linked with disease and health-related phenotypes (Patel 2017).

\section{The Eco-Exposome}

The external contribution to the human exposome is determined by environmental exposure, also termed the "ecoexposome", including exposure via air, food, water, dust, and use of consumer products [reviewed in Escher et al. (2017)]. In 2012, the term was defined by the National Research Council (NRC) of the US National Academy of Sciences. In this report, the committee decided to use a focused definition of exposure science [National Research Council (NRC) 2012]: "Exposure science is defined by this committee as the collection and analysis of quantitative and qualitative information needed to understand the nature of contact between receptors (such as people or ecosystems) and physical, chemical, or biologic stressors. Exposure science strives to create a narrative that captures the spatial and temporal dimensions of exposure events with respect to acute and long-term effects on human populations and ecosystems." The eco-exposome was first discussed by Lioy and Smith (2013), who stressed that "Exposure science includes the core elements of the field and provides a conceptual framework that identifies and links sources of stressors, environmental intensity, time-activity and behavior, stressors and receptors, and outcome of contact." The authors conclude in their 2013 paper: "Concurrently, the next generation of exposure scientists needs to be trained to implement the vision and embrace and quantitatively elaborate on the concept of the eco-exposome. Such an approach can be used to examine and solve human and environmental health problems around the world."

As a variant of the eco-exposome, the concept of the "infectome" was introduced, referring to the collection of an individual's exposures to infectious agents (Bogdanos et al. 2013a, b). The authors describe this part of the exposome as a collection of an individual's exposures to infectious agents participating in the pathogenesis of autoimmune disease (ADs). In this context, infectious agents such as viruses and bacteria can be regarded as environmental triggers that contribute to diseases (Bogdanos et al. 2013b). The infectome is also discussed as a part of the microbiome (that may be regarded as an endogenous exposome) (Bogdanos and Sakkas 2017). Notably, the discussion of the microbiome as part of the exposome was extended by addressing the gastrointestinal exposome representing the integration of all xenobiotic components and host-derived endogenous components affecting the host health, disease progression, and ultimately clinical outcomes during the lifespan (Moon 2016). In this discourse, the microbiomes are considered to reciprocally altering the bioavailability and activities of the chemical exposome in the mucosa, whereas the chemical exposome derived from nutrients and other xenobiotics can influence the dynamics of microbiome community (the stability, diversity, or resilience). The integration of this mucosal crosstalk in the exposome is being discussed as to determining the fate of the microbiome community and host response to the etiologic factors of disease.

Moreover, the eco-exposome has been specified as part of the "blood exposome," which is defined to include all biologically active endogenous and exogenous chemicals circulating in the blood (Rappaport et al. 2014). This interesting approach recognizes that "meaningful exposures are mediated in the internal chemical environment by endogenous signaling molecules, exogenous chemicals, and reactive electrophiles that communicate with cells, tissues, and organs via mutations, posttranslational modifications, enzymes, transcription factors, and receptors (G-factors)."

The authors conclude: "Perhaps the most compelling reason for embracing the blood exposome is the potential to discover all chemicals that cause disease and then to intervene in order to modify exposures and the concomitant burden of disease."

\section{The Endogenous Exposome}

The endogenous exposome refers to internal environmental exposure causing chronic pathologies. As a prominent example of an endogenous exposome, endogenous DNA damage arising from intracellular oxidative stress was recently competently described [for more details (see Nakamura et al. 2014)]. This example was explicitly chosen by the authors because it provides quantitative data on endogenous DNA damage and its relationship to mutagenesis resulting in cancer.

A variant of an endogenous exposome based on oxidative stress can be seen in the radical-free theory of aging [for review, see Go and Jones (2017)]. As concluded in this review by the authors, "the redox theory of aging reveals a 
need to elucidate the central principles of exposure memory, i.e., the central guidelines for lifelong exposures to optimize an individual exposome for healthy longevity.... Simple principles of the redox code are followed in redox organization and function, and the resulting redox networks and exposure memory systems effectively account for the hallmarks of aging."

As recently proposed (Colomina et al. 2018), the "psychoexposome" may be included in endogenous exposomes, at least in a broader sense. However, as discussed in the article, the terms psychology, psychiatry, and neurological diseases are scarce in the exposome approach. Nevertheless, the authors concluded that "psychology may take advantage from both exposome and "omic" sciences to create an integrated psychoexposome approach that may help in deciphering the etiology of psychological disorders and improving people's mental health."

\section{Résumé}

There is much hope that research on the exposome will considerably contribute to the understanding of chronic disease development. Indeed, it is believed that exposomics could have a broad impact on personalized preventative medicine, policy changes, and revealing disease mechanisms since it can be performed at the individual as well as the population level. In particular, exposomics is expected to be used in the context of cumulative risk assessment. Thus, as argued by Smith et al. (2015), “... Exposomics allows us to study interactions between chronic stress and environmental chemicals and to discover environmental chemicals that may disrupt stress response pathways. We have named such chemicals 'stressogens' as they have the ability to influence how our bodies respond to stress."

As will be outlined below, this statement of experts strikingly reflects a considerable interconnectedness between research on exposomics and DAMP-induced innate immune pathways.

\section{The Adverse Outcome Pathway (AOP) Concept}

\section{Introductory Remarks}

The AOP concept has recently been accurately formulated by Leist et al. (2017): "Adverse outcome pathways (AOPs) are a recent toxicological construct that connects, in a formalized, transparent and quality-controlled way, mechanistic information to apical endpoints for regulatory purposes ... in toxicology and ecotoxicology. AOP links a molecular initiating event (MIE) to the adverse outcome (AO) via key events (KE), in a way specified by key event relationships (KER)".
The model is now regarded as a supportive paradigm shift in regulatory toxicology testing and risk assessment. In fact, it is believed that AOPs based on available information on the sequence of events (MIE $\rightarrow \mathrm{KEs} / \mathrm{KERs} \rightarrow \mathrm{AO}$ ) allow the development of relevant predictive animal-free in vitro test methods, as well as the contextualization of the results obtained across a diverse range of biological mechanisms and toxicity endpoints. The relationship of such an AOP concept to the exposure concept can be seen in the narrative level linking exposure to the triggering of an MIE.

According to the Organization for Economic Cooperation and Development (OECD) guidance (OECD 2018), each AOP should have one MIE and one AO, but there is no limitation in the number of KEs. Notably, the OECD represents an international hub for constructing, reviewing, and using AOPs with the help of a suite of tools comprising the AOP knowledge base, including the AOP Wiki (Aopwiki 2020). This AOP Wiki provides a collaborative platform for constructing AOPs and can be used by groups that have a proposal for an AOP (OECD 2018).

Historically, the concept was developed by Ankley et al. (2010), aiming to serve as a reliable parameter in assessing the risk of distinct chemicals, as based on an understanding of the responsible mechanisms in question. Today, the AOP concept is considered as a model that links the exposure of chemicals to their cellular concentrations and MIEs, through KEs to responses at many levels of biological organization, that is, at the subcellular/cellular, organ, organism and, finally, population and even ecosystem level. Of note, with this interpretation in mind, an AOP does not necessarily claim to provide a comprehensive and detailed description of the exact molecular mechanism of action of each chemical concerned.

During the past 10 years, the concept has attracted a large amount of attention, as documented by an increasing number of competent review articles [also see Leist et al. (2017), Villeneuve et al. (2014), Becker et al. (2015), Burden et al. (2015), Perkins et al. (2015), Edwards et al. (2016), OECD (2014) Vinken et al. (2017), Spinu et al. (2020) and Sasaki et al. (2020)]. A few important aspects are described in the following section.

\section{Outline of the Adverse Outcome Pathway Concept}

As mentioned partially above, an AOP alludes to a sequela of measurable events linking a stressor-induced MIE to a final measurable $\mathrm{AO}$ with relevance to risk assessment-via a cascade of KEs while establishing causal links between adjacent KEs, the KERs. The AOPs are chemical-agnostic in that they describe a toxicological process from a purely dynamic, biological perspective. Thus, an AOP can be ultimately associated with any chemical that is bioavailable at 
the relevant site of action and which has the specific features to activate the associated MIE.

Accordingly, in the MIEs, a chemical interacts with a biological target; the following intermediate KEs on the various levels of biological organization are then defined as changes in biological state, which have to be both measurable and essential for the specific AO. Such KEs are believed, for example, to be alterations of a metabolic pathway, signaling events as well as modifications of organelles or cell functions. The scientifically based relationship of KEs refers to the KERs, which define the connection between two adjacent KEs by identifying one as an upstream and the other one as a downstream event. In other words: AOPs span many levels of biological organization, from molecular over subcellular/ cellular to organ and organism or, especially in the case of ecotoxicology, even to the population level. While the MIE always is a molecular-level interaction, the final anchoring point is the AO that is relevant for the entire organism (for example, clinical symptoms defining a typical disease) and represents a classical apical endpoint as used traditionally for hazard evaluation and risk assessment.

Plausibly, this scenario is independent of any specific chemical. However, a chemical may trigger a specific AOP by eliciting the MIE; alternatively, it may be applied to provide empirical evidence for the existence of an AOP. Again, in this sense, AOPs are simplified pragmatic conceptual constructs, defined as linear, non-branching, and directed sequelae of KEs, connecting a single MIE to an AO [for reviews and competent articles, see Leist et al. (2017), Villeneuve et al. (2014), Becker et al. (2015), Burden et al. (2015), Perkins et al. (2015), Edwards et al. (2016), OECD (2014) and Vinken et al. (2017)].

An impressive example of an AOP has been described by Horvat et al. (2017), who chose an AOP development from protein alkylation to liver fibrosis. In the authors' study, MIE refers to protein alkylation. Alkylated proteins can disturb the cellular redox balance in exposed cells by interacting with glutathione, which leads to a disruption of a plethora of biochemical pathways and intracellular stress that, depending on the extent of mitochondrial involvement, can lead to apoptotic or necrotic cell death. As the first KE, hepatocyte death is chosen, the KER between protein alkylation (=MIE) and hepatocyte death $\left(=\mathrm{KE}_{1}\right)$ being discussed as the result of several pathways, including stress responses and lipid peroxidation. Other KEs proposed on the cellular level include Kupffer cell activation, transforming growth factorbeta1 (TGF- $\beta 1$ ) expression, stellate cell activation, as well as collagen accumulation and changes in extracellular matrix (ECM) composition on the tissue level. Finally, liver fibrosis affecting the whole liver was chosen to serve as the AO.

\section{Résumé}

As stressed by Leist et al. (2017), the AOP concept represents a crucial research opportunity and has assisted in integrating molecular mechanisms into the field of regulatory toxicology. For the future, AOP application is expected to serve as an AOP-based hazard assessment based on in vitro MIE and KE testing, an approach that is supposed to replace the current toxicological methodologies. To quote the authors (Leist et al. 2017): "A dedicated research program to specifically confirm and validate AOPs and to add quantitative information on KERs is required. There is however an expectation that the AOP conceptual framework would help in pushing through a mechanistic shift in risk assessment by making use of all available information in a weight of evidence approach and/or forming the basis for a mechanistically driven testing strategy. In the short term, it is also expected that the framework will be used as a scaffold for developing guidance for complex hazard characterization, providing a mechanistic contextualization of apical end points of concern. And the authors state: ... What is required is individual KEs with a high plausibility value (with respect to a link to an AO) that can be studied in vitro in a concentration-dependent manner. Examples are compromised migration of neuronal precursors or compromised mitochondrial functions."

However, hard data to realize this ambitious goal still lack in this field of research. Perhaps, part of this gap can be filled by the introduction of DAMPs as an integral part of this concept - a proposal that will be further discussed in the following sections.

\section{DAMPs as Major Integral Part of the Exposome and AOP Concepts}

\section{Introductory Remarks}

In light of the danger/injury model in Immunology, it is tempting to integrate DAMP-promoted innate and adaptive immune responses-in terms of uncontrolled, dysregulated responses - in both the exposome and the AOP concept. Accordingly, the goal of "DAMPomics" research would be to measure all exogenous and endogenous DAMPs [as summarized in Land (2018), Chpts. 11-16], which are involved in promoting innate immune effector responses and adaptive immune processes. Taking into account the definition of the exposome and the AOP concept as briefly mentioned above, a tentative scenario of four major events may be sketched (Fig. 2): 


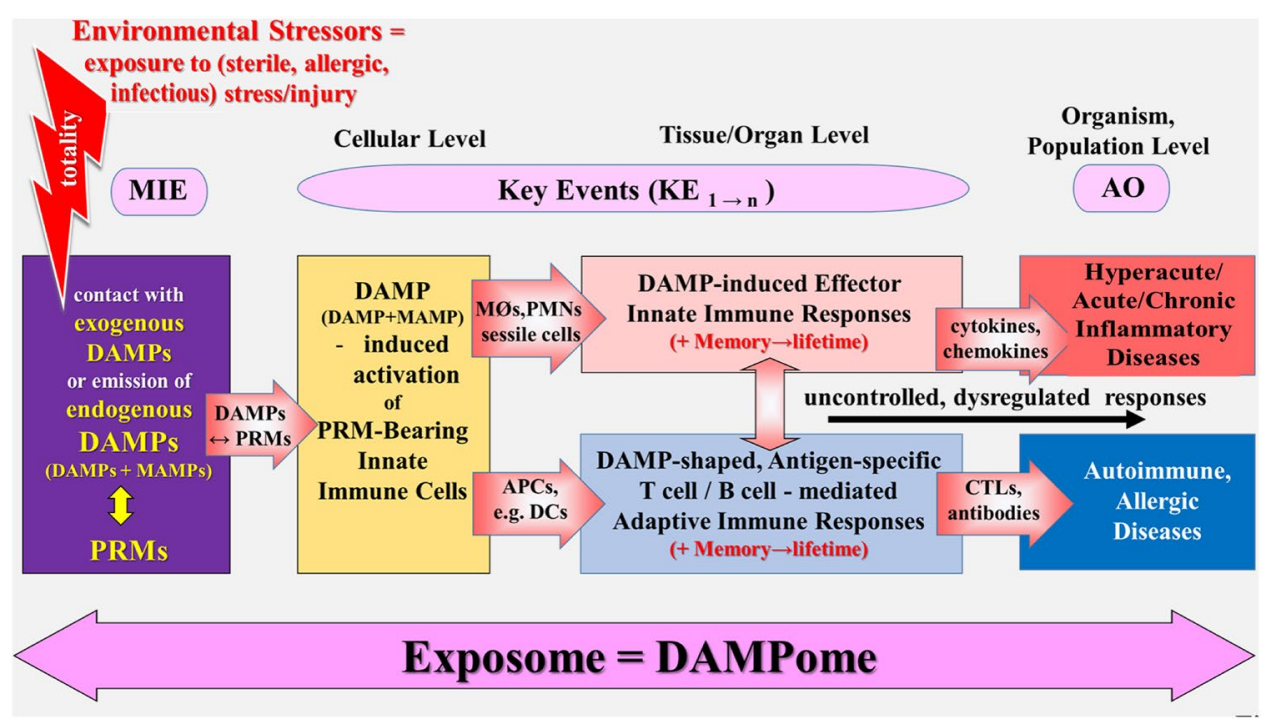

Fig. 2 Schematic representation of a simplified narrative scenario model of integrating DAMP-promoted innate and adaptive immune responses (in terms of uncontrolled, dysregulated responses) in both the exposome and the AOP concept. Any infectious or sterile stress/injury (i.e., environmental stressors) are mediated (1) directly by exogenous DAMPs, or (2) indirectly by stress/injury-induced endogenous DAMPs, which interact with PRMs on or in cells of the innate immune system (in the case of infectious injury, together with MAMPs). Following recognition of DAMPs (plus MAMPs, respectively), PRM-bearing cells get activated to trigger innate immune inflammatory responses or, in the presence of nonself or altered-self antigens, to promote antigen-specific adaptive immune responses. DAMP-triggered innate immune effector responses, when uncontrolled and dysregulated, result in pathologies such as acute/chronic

- Any stress/injury, even the slightest molecular perturbation,- -in terms of external and internal environmental stressors - either is mediated by injurious molecules that intrinsically function as exogenous DAMPs or leads to the emission of endogenous DAMPs, both categories of DAMPs interacting with (being sensed or recognized by) PRMs on or in cells of the host's innate immune system (=MIE). In the case of infectious stress/injury, these PRMs also sense/recognize microbe-associated molecular patterns (MAMPs), also called pathogen-associated molecular patterns (PAMPs).

- Following recognition of DAMPs, PRM-bearing cells of the innate immune system get activated $\left(=\mathrm{KE}_{1}\right)$ and promote further events $\left(\mathrm{KE}_{1 \rightarrow n}\right)$.

- As mentioned above, MAMP/DAMP-activated, PRMbearing innate mobile cells and/or sessile cells promote cellular/humoral innate immune effector responses such as inflammation and phagocytosis; in the presence of bacterial/viral antigens, altered-self antigens, allergens, or alloantigens, MAMP/DAMP-activated, PRM-bearing innate antigen-presenting-cells (APCs) such as DCs initiate and elicit adaptive immune responses. (auto)inflammatory diseases, DAMP-shaped, antigen-specific adaptive immune responses, when uncontrolled and dysregulated, can lead to immune pathologies such as autoimmune or allergic diseases. Totality: all environmental chemicals acting as stressors ("stressogens") on a particular receptor or biological pathway in a functional assay [according to Smith et al. (2015)]. AO adverse outcome, AOP adverse outcome pathway, APCs antigen-presenting cells, CTLs cytotoxic T lymphocytes, DAMPs damage-associated molecular patterns, $D C s$ dendritic cells, $K E$ key event, $M A M P s$ microbe-associated molecular patterns, $M I E$ molecular initiating event, $M \emptyset$ macrophages, PMNs polymorphonuclear neutrophils, PRMs pattern recognition molecules. Sources: Smith et al. (2015), Escher et al. (2017), Leist et al. (2017) and Vinken et al. (2017)

- As also touched above, DAMP-promoted cellular/ humoral innate immune effector responses primarily serve maintenance and restoration of homeostasis; however, when uncontrolled and dysregulated, they result in pathologies such as hyperacute, acute, or chronic (auto) inflammatory diseases (=AO). DAMP-shaped, antigenspecific adaptive immune responses-when uncontrolled and dysregulated-lead to immune pathologies such as autoimmune or allergic diseases $(=\mathrm{AO})$. Both uncontrolled processes can mutually aggravate the clinical symptoms of the disorders.

The whole process starting from exposure to a distinct environmental (infectious/noninfectious) stress/injury (stressor), associated with the presence/emission of DAMPs, up to the manifestation of a disease may be regarded as an exposome. In the following, a few details of such a scenario are more closely looked at. 


\section{DAMPs as an Integral Part of the Exposome Concept}

\section{From the Exposome to the "DAMPome"}

Stress/injury-induced, DAMP-triggered, PRM-meditated efferent innate immune and DAMP-shaped antigen-specific adaptive immune events are processes that may be regarded as part of the exposome concept and represent another effort to advance exposome research. A first approach to this notion may be the tentative translation of some passages from the article of Smith et al. (2015) into the "DAMP language":

Original text: "Under the exposome paradigm all nongenetic factors contributing to disease are considered to be 'environmental' including chemicals, drugs, infectious agents and psycho-social stress. We can consider these collectively as environmental stressors. Exposomics is the comprehensive analysis of exposure to all environmental stressors and should yield a more thorough understanding of chronic disease development. We can operationalize exposomics by studying all the small molecules in the body and their influence on biological pathways that lead to impaired health.

Here, we describe methods by which this may be achieved and discuss the application of exposomics to cumulative risk assessment in vulnerable populations. Since the goal of cumulative risk assessment is to analyze, characterize, and quantify the combined risks to health from exposures to multiple agents or stressors, it seems that exposomics is perfectly poised to advance this important area of environmental health science. ..... Thus, through linking exposures to specific biological responses, exposomics could serve as an approach to gain insight into the mechanistic connections between a culmination of exposures and risk of adverse health outcomes that occur over a lifetime."

Tentative Translation into the "DAMP language" Under the "DAMPome" paradigm, all non-genetic factors contributing to disease are considered to be environmental factors including chemicals, drugs, infectious agents, and psychosocial stress. We can consider these collectively as "DAMPs". They are denoted as exogenous DAMPs if the stress/injurymediating molecules intrinsically function as DAMPs; they are called endogenous DAMPs if they are emitted upon sterile, allergic, or infectious stress/injury in the host's body.

"DAMPomics" is the comprehensive analysis of exposure to all exogenous and endogenous DAMPs and should yield a more thorough understanding of both acute and chronic disease development. We can operationalize "DAMPomics" by studying all the MAMP and/or DAMP-induced, PRMtriggered signaling molecules in the body and their influence on eliciting efferent (cellular/humoral) innate immune and adaptive immune pathways $\rightarrow$ responses that - when controlled-lead to homeostasis, - when uncontrolled and dysregulated - to pathologies and diseases.

Here, we describe methods (to measure in vitro and in vivo MAMP and/or DAMP-induced PTM-triggered innate or DAMP-shaped adaptive immune responses) by which this may be achieved and discuss the application of "DAMPomics" to cumulative risk assessment in vulnerable populations. Since the goal of cumulative risk assessment is to analyze, characterize, and quantify the combined risks to health from exposures to multiple (distinct patterns of) DAMPs, it seems that "DAMPomics" is perfectly poised to advance this important area of environmental health science. ...... Thus, through linking exposures to specific DAMP-promoted innate/adaptive immune responses, "DAMPomics" could serve as an approach to gain insight into the mechanistic connections between a culmination of exposures to exogenous and endogenous DAMPs and risk of pathologies and diseases that occur over a lifetime. Such a mechanistic connection between culmination of exposures and risk of diseases that occur over a lifetime may be implicated by the phenomena of innate immune memory ("trained immunity") (Netea et al. 2016) and adaptive immune memory [for immune memory, see also Land (2018) Chpt. 24, p. 635 and Chpt. 32, p. 749].

\section{The Puzzle of Environmental Factors in Relation to Exogenous and Endogenous DAMPs}

As mentioned above, the exposome paradigm includes exposure to external environmental factors [eco-exposome (Lioy and Smith 2013)] and internal environmental factors (endogenous exposome (Nakamura et al. 2014)). This differentiation also needs a tentative translation. In fact, in principle, external environmental factors may directly act as exogenous DAMPs or indirectly - as internal environmental factors derived from exogenous sources-induce emission of constitutive/inducible endogenous DAMPs in the host. On the other hand, internal environmental factors derived from endogenous sources (such as the generation of ROS) induce notoriously emission of constitutive/inducible endogenous DAMPs. Examples of such a translation procedure are:

- Some reactive electrophilic compounds such as aldehydes, alkenals, and tear gases are exogenous DAMPs which - in the sense of external environmental factorsinduce nociceptor-triggered inflammatory pain pathways [see Land (2018), Chpt. 15, p. 353];

- UV radiation is an external environmental factor that induces endogenous DAMPs which activate PRM-bearing skin cells such as keratinocytes (e.g., see Hudson et al. 2020); 
- Aluminum salts, asbestos fibers, silica particles, and quartz dust are exogenous DAMPs, which-in the sense of internal environmental factors derived from exogenous sources-induce PRM-triggered, NLRP3 inflammasome-mediated innate immune responses [see Land (2018), Chpt. 15, p. 353 and Chpt. 22, p. 475, as well as Hedbrant et al. (2020) and Dai et al. (2020)].

- Allergens-in the sense of external environmental factors-may directly act as exogenous DAMPs such as some metal allergens (e.g., nickel) (see Land (2018), Chpt. 15, p. 353) or, for examples, as proteases-in the sense of internal environmental factors derived from exogenous sources-may induce constitutive and inducible endogenous DAMPs such as ATP, uric acid, and IL-1 family members (Land 2018; Willart et al. 2013; Ramu et al. 2018).

- Oxidative stress, for example, induced by postischemic tissue reperfusion (IRI) is an internal environmental factor derived from endogenous sources that induces endogenous DAMPs [see Land (2018), Chpt. 13, p. 269 and Chpt. 19, p. 427];

- Psychological stress is an internal environmental factor derived from endogenous sources that induces endogenous DAMPs such as HSP72 (Fleshner et al. 2004).
These few examples should provide sufficient evidence in support of the notion that definitions and criteria of the exposome paradigm match well with the concept of a "DAMPome". In fact, in line with such an argumentation, DAMPs may operate as true signatures of (external/internal) environmental exposure.

\section{DAMPs as an Integral Part of the AOP Concept}

\section{General Remarks}

Given the current state-of-the-art in AOP research as briefly summarized above, it appears to be attractive to include the DAMPs in in vivo and in vitro approaches to optimize AOPbased hazard assessment. Interestingly, a first such attempt has already been reported by van Bilsen et al. (2017), who discussed the application of the AOP concept aiming "to structure the available in vivo and in vitro mechanistic data for allergic sensitization to food proteins." More generalized speaking, in vitro measurement of DAMPs together with their cognate PRMs, and subsequently triggered activation of cells of the innate immune system would make sense as an amendment of in vitro MIE and KE testing. However, an important note has to be made: the double role of DAMP-induced immune pathways. Thus, when controlled and adequately regulated, DAMP-induced pathways serve

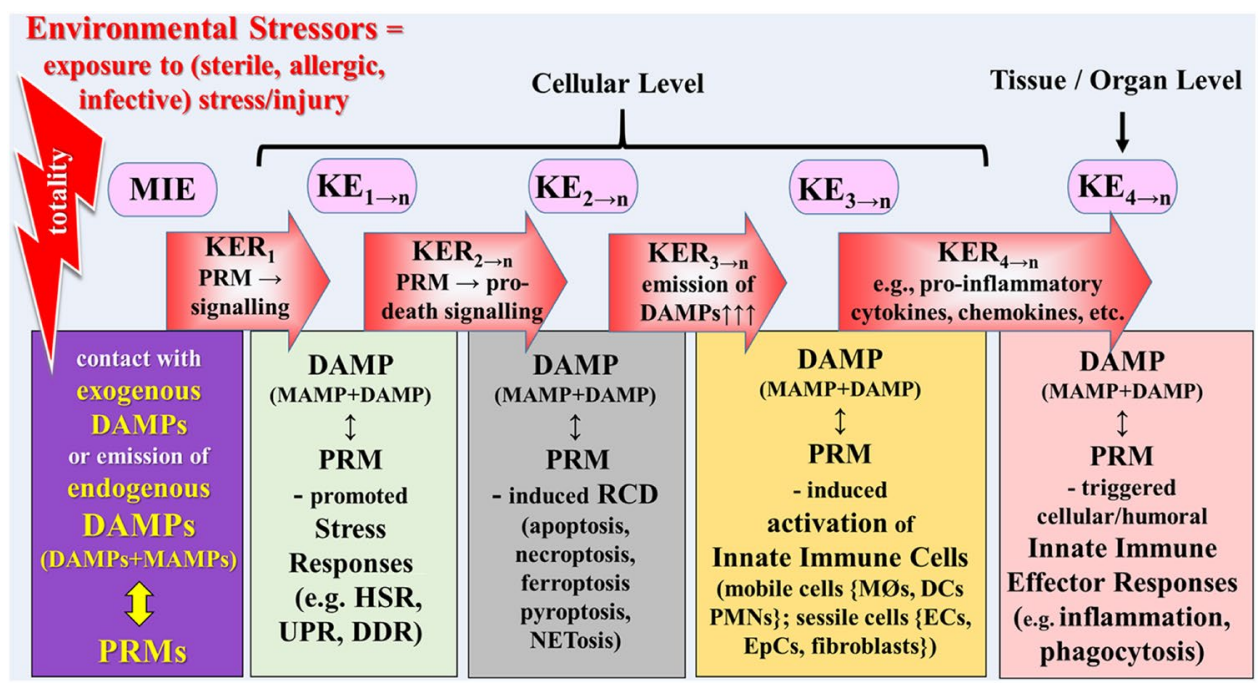

Fig. 3 Schematic representation of a narrative scenario model of controlled homeostatic DAMP-promoted pathways in light of the exposome and AOP concepts. The figure shows the first part of key events (KEs). The initiating event expressed by DAMPs $\leftrightarrow$ PRMs interaction (MIE) leads to a sequential cascade of cellular, tissue, and organ responses (key events $=\mathrm{KEs}$ ), linked to each other by key event relationship (KER). DAMP-promoted, failing (!) stress responses $\left(\mathrm{KE}_{1-n}\right)$ result in subroutines of regulated cell death $\left(\mathrm{KE}_{2-n}\right)$ that are associated with release of DAMPs. DAMPs (together with MAMPs in case of infectious injury) are sensed by PRMs to trigger activation of innate immune cells $\left(\mathrm{KE}_{3-n}\right)$, which trigger the fourth key event in terms of innate immune inflammatory responses. $A O$ adverse outcome, $A O P$ adverse outcome pathway, DAMPs damage-associated molecular patterns, $D C s$ dendritic cells, $D D R$ DNA damage response, $E C s$ endothelial cells, EpCs epithelial cells, HSR heat shock response, $K E$ key event, KER key event relationship, MAMPS microbe-associated molecular patterns, $M I E$ molecular initiating event, $M \emptyset$ macrophages, $P M N s$ polymorphonuclear neutrophils, $P R M s$ pattern recognition molecules, $R C D$ regulated cell death, $U P R$ unfolded protein response. Sources: Smith et al. (2015), Escher et al. (2017), Leist et al. (2017) and Vinken et al. (2017) 


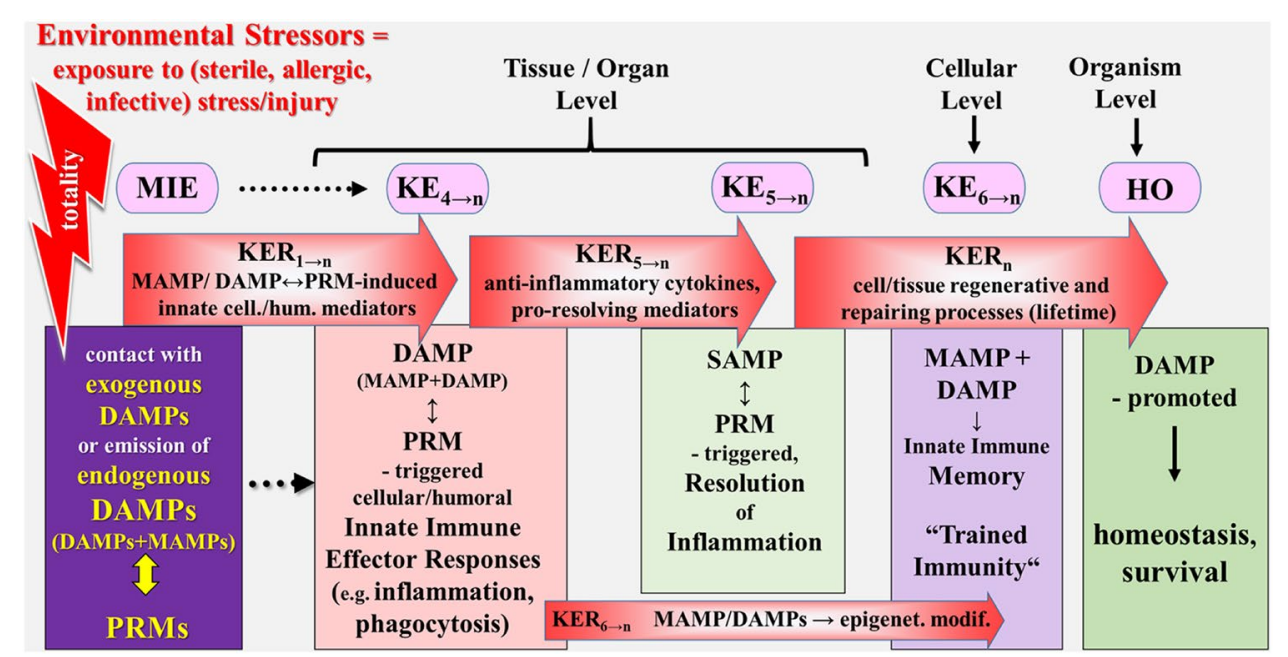

Fig. 4 This figure is the continuation of Fig. 3 and shows the second part of key events resulting in homeostasis, whereby two phases of events (MIE and $\mathrm{KE}_{4 \rightarrow n}$ ) are depicted again. Thus, the fourth inflammatory event proceeds to SAMP-driven resolution of inflammation $\left(\mathrm{KE}_{5-n}\right)$, associated with MAMP/DAMP-promoted induction of an innate immune memory $\left(\mathrm{KE}_{6-n}\right)$, finally resulting in homeostasis.
$D A M P s$ damage-associated molecular patterns, $H O$ homeostasis, $K E$ key event, KER key event relationship, MAMPs microbe-associated molecular patterns, MIE molecular initiating event, $P M N s$ polymorphonuclear neutrophils, PRMs pattern recognition molecules, SAMPs suppressing DAMPs. Sources: Smith et al. (2015), Escher et al. (2017), Leist et al. (2017) and Vinken et al. (2017)

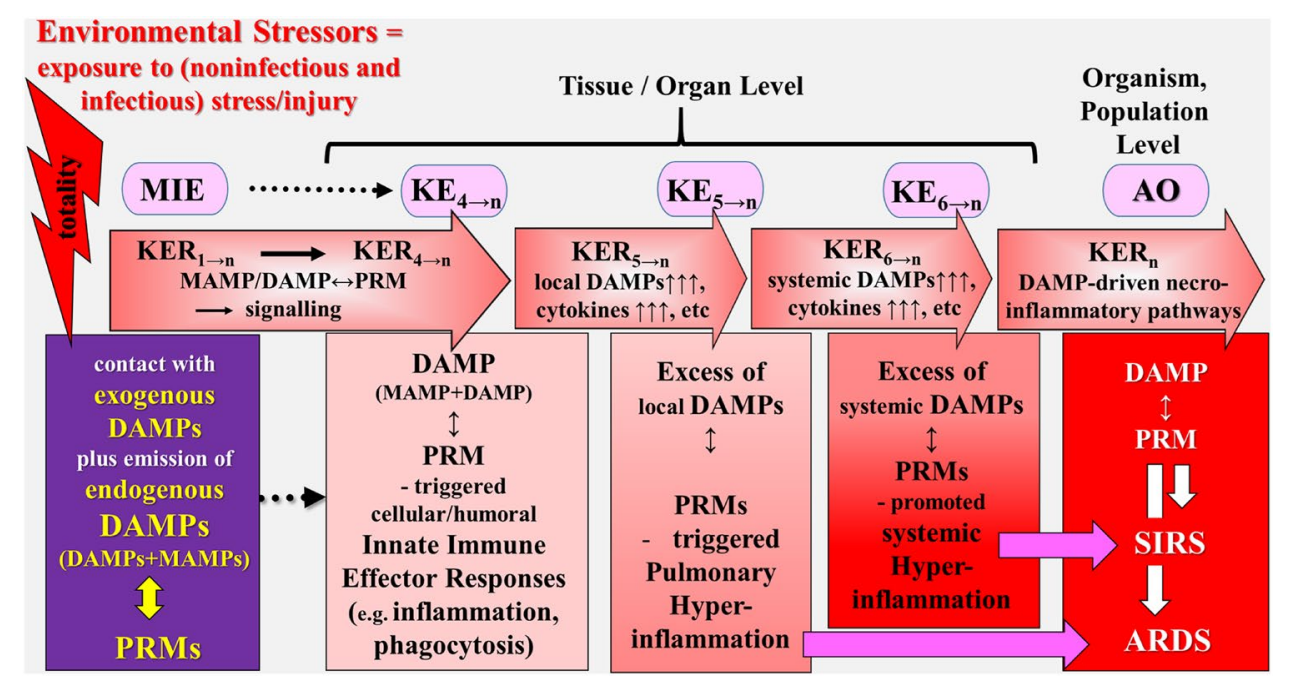

Fig. 5 Schematic representation of a simplified narrative scenario model of uncontrolled dysregulated, DAMP-promoted pathways in light of the exposome and AOP concepts. The figure illustrates the sequelae of key events proposed to leading to ARDS and SIRS, reflecting the adverse outcome in COVID-19. The promoted dysregulated pathways leading to COVID-19 starts with the sequelae of events (MIE $\rightarrow \mathrm{KE}_{4 \rightarrow n}$ ) as described for the controlled pathway (Fig. 3). However, instead of proceeding to homeostatic events, the DAMP-induced pathways now provoke "pathogenic" KEs key events resulting in ARDS and/or SIRS/sepsis. The triggering "aberrant"

to maintain and restore homeostasis; however, when uncontrolled and dysregulated, DAMP-promoted processes result in pathologies and diseases, that is, adverse health outcome. Accordingly, when trying to apply the exposome and AOP event is the fifth KE that refers to pulmonary hyperinflammation, as characterized by intrapulmonary emission of DAMPs in excessive, which may spread out systemically. The adverse outcomes refer to ARDS and/or SIRS/sepsis. AO adverse outcome, ARDS acute respiratory distress syndrome, DAMPS damage-associated molecular patterns, $K E$ key event, KER key event relationship, MAMPs microbeassociated molecular patterns, MIE molecular initiating event, PRMs pattern recognition molecules, SIRS systemic inflammatory response syndrome. Source: Land (2018)

concepts to this scenario, one has to differentiate between controlled homeostatic pathways (Figs. 3, 4) and uncontrolled dysregulated pathways $=$ AOPs (Figs. 5, 6, 7, 8). 


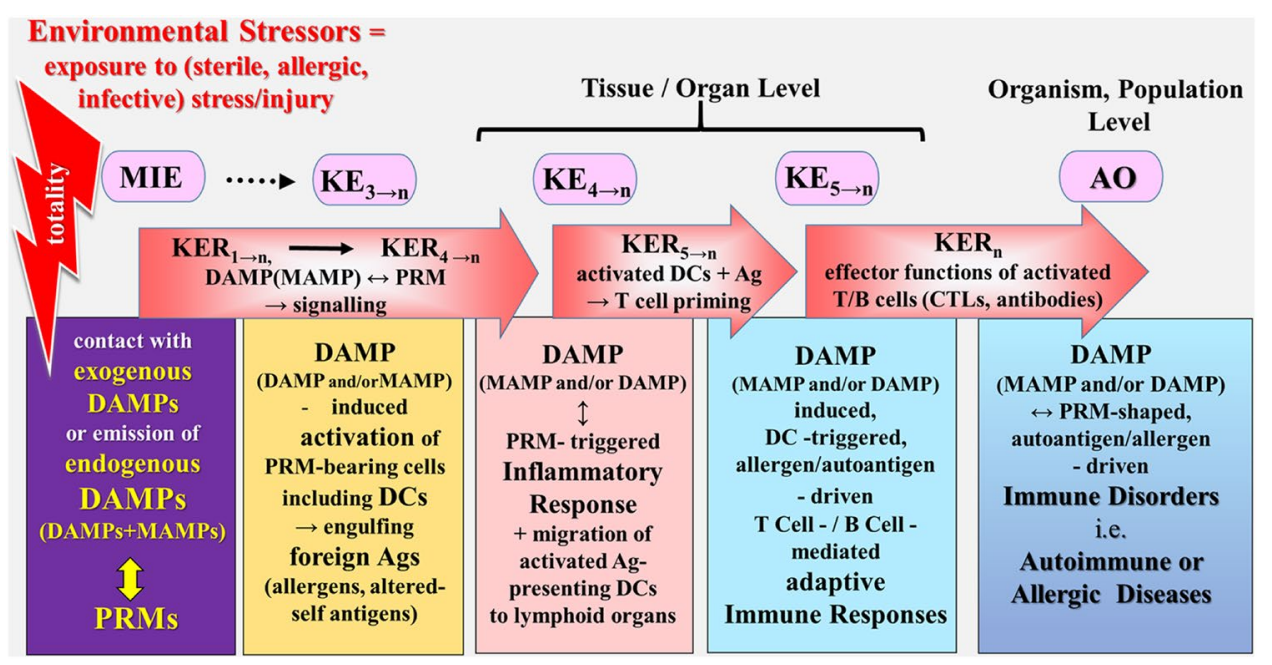

Fig. 6 Schematic representation of a simplified narrative scenario model of uncontrolled dysregulated, DAMP-promoted pathways in light of the exposome and AOP concepts. The figure illustrates the sequelae of key events proposed to leading to immune disorders, reflecting the adverse outcome. The stress/injury $\rightarrow$ DAMPpromoted, dysregulated pathways leading to immune diseases start with the sequelae of events (MIE $\rightarrow \mathrm{KE}_{3 \rightarrow n}$ ) as described for the controlled pathway (Fig. 3). However, instead of proceeding to homeostatic events, the DAMP-induced pathways now provoke "pathogenic" KEs key events resulting in an AO in terms of immune disorders such as autoimmune or allergic diseases. $A g$ antigen, $A O$ adverse outcome, CTLs cytotoxic T cell lymphocytes, DAMPs damage-associated molecular patterns, $D C s$ dendritic cells, $K E$ key event, KER key event relationship, MAMPs microbe-associated molecular patterns, MIE molecular initiating event, PRMs pattern recognition molecules. Sources: Land (2018), Smith et al. (2015), Escher et al. (2017), Leist et al. (2017) and Vinken et al. (2017)

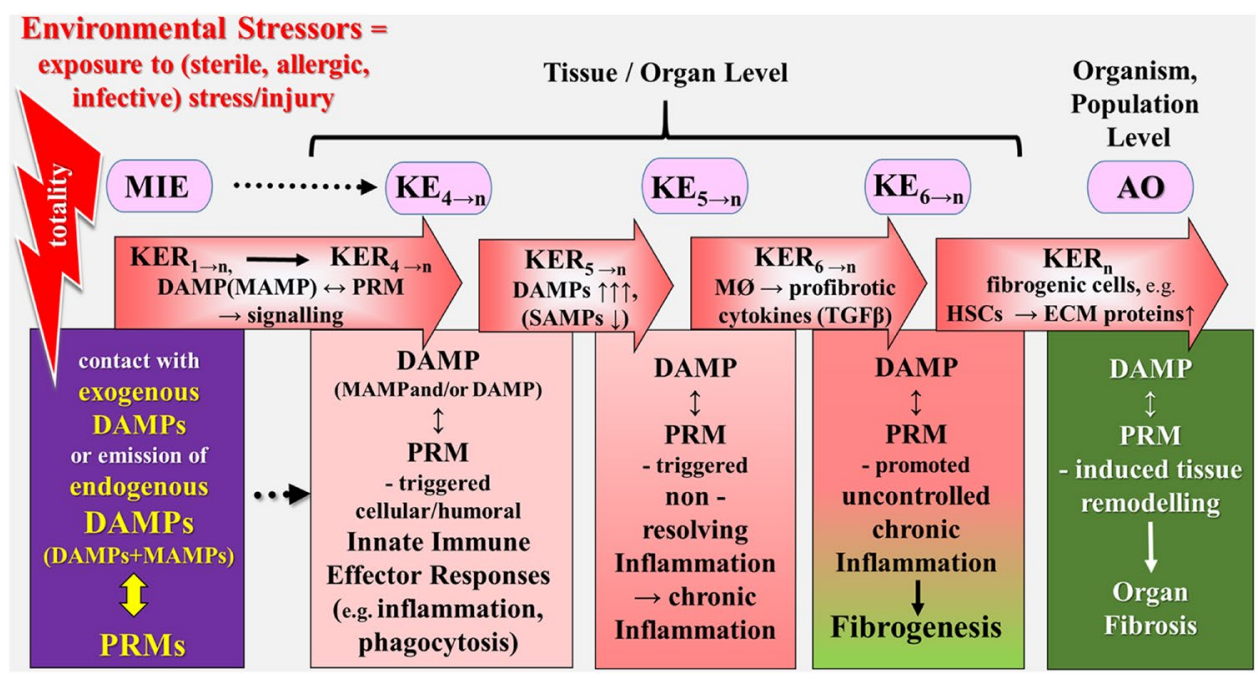

Fig. 7 Schematic representation of a simplified narrative scenario model of uncontrolled dysregulated DAMP-promoted pathways in light of the exposome and AOP concepts. The figure illustrates the sequelae of key events proposed to leading to fibrotic disorders (organ fibrosis), reflecting the adverse outcome. The stress/injury $\rightarrow$ DAMP-promoted dysregulated pathways leading to immune diseases start with the sequelae of events (MIE $\rightarrow \mathrm{KE}_{3 \rightarrow n}$ ) as described for the controlled pathway (Fig. 3). However, instead of proceeding to homeostatic events, the DAMP-induced pathways now provoke "pathogenic" KEs key events resulting in an AO in terms of fibrotic disorders. The triggering "aberrant" event is the fifth KE that refers to (uncontrolled) chronic inflammation as characterized by the failure of an inflammation-resolution response (= "nonresolving inflammation"), proceeding to fibrogenic responses (fibrogenesis). The adverse outcome refers to complete organ fibrosis associated with typical organ-specific clinical symptoms. $A O$ adverse outcome, DAMPs damage-associated molecular patterns, ECM extracellular matrix, HSCs hepatic stellate cells, $K E$ key event, $K E R$ key event relationship, $M A M P s$ microbe-associated molecular patterns, MIE molecular initiating event, $P R M s$ pattern recognition molecules, SAMPs suppressing DAMPs, $T G F-\beta$ transforming growth factor-beta. Source: Land (2018) 


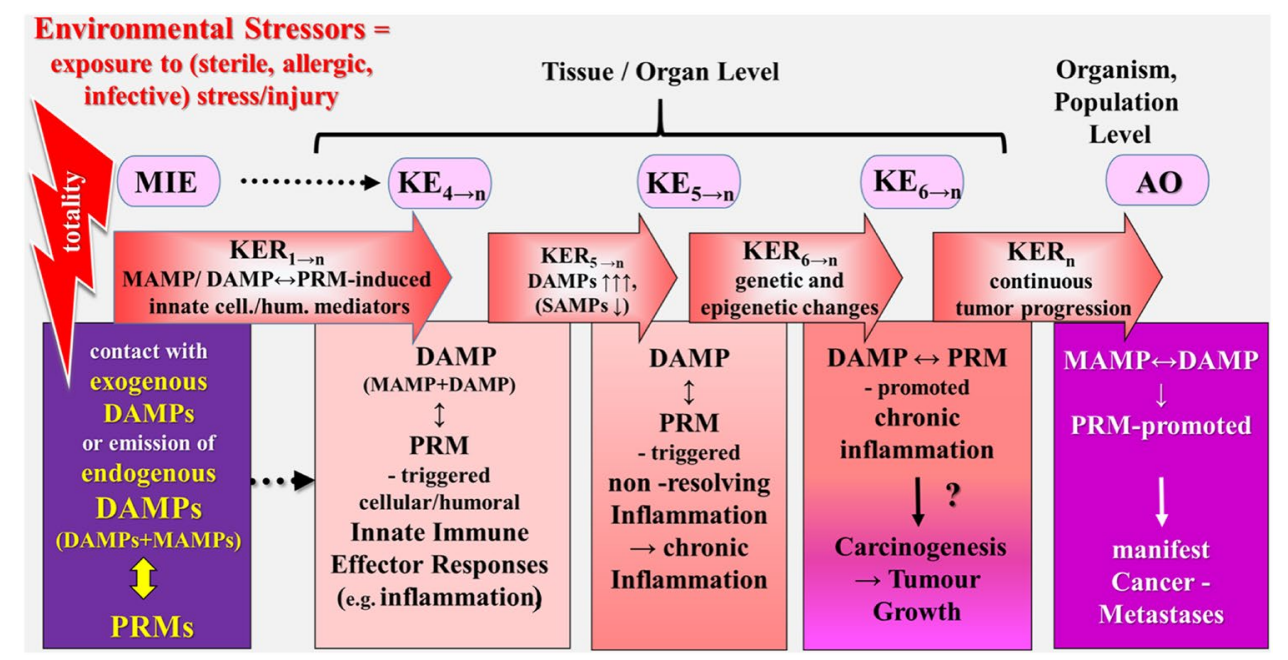

Fig. 8 Schematic representation of a simplified narrative scenario model of uncontrolled dysregulated DAMP-promoted pathways in light of the exposome and AOP concepts. The figure illustrates the sequelae of key events proposed to leading to cancer, reflecting the adverse outcome. The stress/injury $\rightarrow$ DAMP-promoted, dysregulated pathways leading to immune diseases start with the sequelae of events (MIE $\rightarrow \mathrm{KE}_{3 \rightarrow n}$ ) as described for the controlled pathway (Fig. 3). However, instead of proceeding to homeostatic events, the DAMP-induced pathways now provoke "pathogenic" KEs key events resulting in an adverse outcome in terms of cancer. The triggering "aberrant" event is the fifth KE that refers to (uncontrolled) chronic inflammation as characterized by the failure of an inflammation-resolution response. However, the exact mechanisms underlying chronic inflammatory microenvironment-promoted carcinogenesis are not fully understood. $A O$ adverse outcome, cell./hum. cellular/humoral, $D A M P s$ damage-associated molecular patterns, $K E$ key event, $K E R$ key event relationship, MAMPs microbe-associated molecular patterns, MIE molecular initiating event, $P R M s$ pattern recognition molecules, SAMPs suppressing DAMPs. Sources: Balkwill and Mantovani (2001), Hecht (2002), Grivennikov et al. (2010), Ben-Neriah and Karin (2011), Wang et al. (2016) and Zhang and Xu (2017)

RNA-like endoplasmic reticulum kinase; ATM for Ataxiatelangiectasia mutated kinase).

The second major KE reflects the incidence of DAMP/ (MAMP)-induced, PRM-triggered RCD occurring in case of stress response failure due to too severe stress/injury [compare Land (2018), Chpt. 19, p. 427]. Typically, several subroutines of RCD, in particular, necroptosis, ferroptosis, pyroptosis, and NETosis $\left(\mathrm{KE}_{2 \rightarrow n}\right)$, are associated with passive release of large amounts of DAMPs. PRM-triggered pro-death signaling pathways serve as a second KER, resulting, instead of cell survival, in cell death.

The third major KE may point to DAMP/MAMP-induced activation of PRM-bearing innate immune cells such as mobile phagocytic and sessile cells, that is, the process of the sterile/allergic/infectious stress/injury-induced activation of the innate immune system. Since many families of innate immune cells with different functions are involved (including neutrophils, macrophages, DCs, vascular cells, fibroblasts, and epithelial cells), many KEs may be included in this major $\mathrm{KE}\left(\mathrm{KE}_{3 \rightarrow n}\right)$. As a critical example of several potential KERs involved in this transition point, the passive release of DAMPs may be used here $\left(\mathrm{KER}_{3 \rightarrow n}\right)$.

The fourth KE refers to DAMP/MAMP-induced, PRMtriggered, cell-mediated and humoral efferent innate immune 
responses, that is mainly and typically, inflammation and inflammation-associated events such as phagocytosis and complement activation [see Land (2018), Chpt. 22, p. 475 and Chpt. 23, p. 591]. The many facets of those DAMP-promoted innate immune effector processes can be divided into various KEs $\left(\mathrm{KE}_{4 \rightarrow n}\right)$. As KERs, pharmacological mediator substances produced by activated innate immune cells such as cytokines, chemokines, and adhesion molecules, as well as complement fragments were chosen, known to create and orchestrate inflammatory and inflammation-related processes $\left(\mathrm{KER}_{4 \rightarrow \mathrm{n}}\right)$ (also compare Fig. 3).

The fifth major KE considers the process of SAMP-driven resolution of inflammation that reflects the homeostatic aim and final result of inflammation (Fig. 4) [see Land (2018), Chpt. 22, p. 475]. It is an active process of restoration of affected/damaged tissues to their normal structural and functional state ("restitutio ad integrum"). This unique, highly coordinated program, as hauntingly reflected by the phenomenon of wound healing, is governed by innate immune cells and specific mediators produced by them $\left(\mathrm{KE}_{5 \rightarrow n}\right)$. As KERs serve anti-inflammatory cytokines and SAMPS, e.g., pro-resolving mediators [see Land (2018), Chpt.14, p. 307], conveying anti-inflammatory, inflammation-resolving, and immunosuppressive effects $\left(\mathrm{KER}_{5 \rightarrow n}\right)$. The process of resolution of inflammation finally leads to homeostasis (HO) in terms of the survival outcome of the individual.

The six major KE refers to the phenomenon of "trained immunity," reflecting the memory of innate immune responses (Netea and van der Meer 2017). The recent discovery of this phenomenon may allow the application of cumulatively experienced MAMP/DAMP-induced innate immune responses ("DAMPomics"), occurring during whole lifetime to cumulative risk assessment in vulnerable populations. As KERs serve MAMP/DAMP-promoted epigenetic modifications $\left(\mathrm{KER}_{6 \rightarrow n}\right)$. The event of "trained immunity" can be considered as an integral part of host defense contributing to lifetime maintenance of homeostasis.

\section{Uncontrolled DAMP-Promoted Pathways in Light of the Exposome and AOP Concepts: a Few Examples}

General Remarks Uncontrolled dysregulated DAMP-promoted pathways resulting in an AO that is, a human disease, pass through the initial events (MIE to $\mathrm{KE}_{4 \rightarrow n}$ ) as shown for controlled pathways (Fig. 4). At this point, however, the pathway can turn into an AOP instead of proceeding to homeostatic events. Such a scenario, for example, can be caused by an uncontrolled aggravation of inflammation to a state of hyperinflammation, promoted by overshooting dysregulated emission of DAMPs. Clinically, this event is presented as a SIRS. A further accident can be caused by the presence of harmful foreign antigens such as allergens or stress/injury-induced altered-self antigens (autoantigens) that act together with DAMPs to shape a specific adaptive immune response. Another disturbance at this point is the perpetuation of inflammation leading to chronic inflammation. Notably, chronic inflammation can be caused by either (1) a failure of the inflammation-resolution response caused by insufficient generation of SAMPs (non-resolving inflammation regarded as a major driver of diseases), or by (2) persistent/acutely repetitive emission of DAMPs, or even by (3) both processes [see Land (2018), Chpt. 22, p. 475]. Here, four typical exemplary models related to these accidents should be briefly touched.

Superimposition of Noninfectious Environmental and Infectious Factors Promote SARS case Fatality: Sketching a Model In general, epidemics caused by viruses such as SARS-CoV and the newly emerging SARS-CoV-2 occur during the winter months. Indeed, as recently reviewed (Moriyama et al. 2020), the major contributing factor (besides human behavior) to respiratory virus outbreaks are the changes in environmental parameters. On the other hand, however, thermal stress/injury, either cold or heat, has been experimentally shown to be associated with emission of endogenous DAMPs (Cai et al. 2019; Liu et al. 2019).

Likewise, other environmental factors may operate directly as exogenous DAMPs in respiratory tract inflammation such as inhaled airborne PM, pathogenic air pollutants, including asbestos and crystalline silica [reviewed in Land (2018), Chpt. 15, p. 353]. This category of DAMPs was shown to trigger activation of the NLRP3 inflammasome associated with inflammasome-dependent proinflammatory processes (Moloudizargari et al. 2019; Dai et al. 2020) as well as, even more important, RCD in form of pyroptosis known to be associated with release of endogenous DAMPs [reviewed in Sarhan et al. (2018)].

In this context of considerable interest are observations from a study on adult New York State residents revealing that increased concentrations of fine PM air pollution of $2.5 \mathrm{~mm}$ or less in diameter are associated with increased rates of respiratory infections, including influenza (Croft et al. 2019). Even of intriguing interest are earlier reports of Chinese groups on a possible link between environmental factors and SARS case fatality (Kan et al. 2005; Cui et al. 2003; Sun et al. 2020). Of special importance are two studies, which had already demonstrated a positive association between pollutants and SARS case fatality in Chinese populations (Kan et al. 2005; Cui et al. 2003). With this background, Qu et al. (2020) recently argued: "The high levels of PM pollution in China may increase the susceptibility of the population to more serious symptoms and respiratory complications of the disease. ... The simultaneous inhalation of chemical pollutants in PM alongside COVID-19 virus may also exacerbate the level of COVID-19 infection. Pro-inflammation, injury, and fibrosis from inhaled PM combined with 
an immune response or cytokine storm induced by COVID19 infection could enhance the infection severity." Remarkably, this suggestion has quite recently been confirmed: A Harvard University environmental research study could demonstrate that a small increase in long-term exposure to fine particulate matter $\left(\mathrm{PM}_{2.5}\right)$ results in a large increase in COVID-19 death rate, with the magnitude of increase 20 times that observed for $\mathrm{PM}_{2.5}$ and all-cause mortality (Wu et al. 2020). According to the investigators' conclusion, the study data underline the importance of continuing to enforce existing air pollution regulations to protect human health both during and after the COVID-19 (Wu et al. 2020). Of note, results of a recent Chinese study could confirm these observations showing that there is a significant relationship between air pollution and COVID-19 infection (Zhu et al. 2020). The authors concluded that this data "could partially explain the effect of national lockdown and provide implications for the control and prevention of this novel disease." Similarly, a Northern Italy environmental study provided evidence suggesting that people living in an area with high levels of pollutants are more prone to develop chronic respiratory conditions (Conticini et al. 2020). Moreover, in this analysis, prolonged exposure to air pollution was found to lead to a chronic inflammatory stimulus, even in young and healthy subjects. The authors concluded that the high level of pollution in Northern Italy (as a lowland) should be considered an additional co-factor of the high level of SARS-CoV-2 lethality recorded in that area (Conticini et al. 2020). In support of such a concept would also be epidemiological data suggesting that, in turn, living in high-altitude may protect from severe impact of acute SARS-CoV-2 virus infection (Arias-Reyes et al. 2020).

On the other hand, in respiratory virus infections, emission of endogenous DAMPs, for example, released via virus-induced RCD (e.g., pyroptosis, necroptosis), has been demonstrated to promote a robust antiviral inflammatory response (Patel et al. 2018) (Nailwal and Chan 2019). In fact, SARS-CoV has already been shown-and SARS CoV-2 is suggested - to induce pyroptotic and necroptotic cell death (Chen et al. 2019b; Yang et al. 2020; Yue et al. 2018), known, as mentioned, to release large amounts of endogenous DAMPs (Sarhan et al. 2018). Consequently, available pharmaceutical agents that target a critical component of the inflammasome-pyroptotic pathway have already recently been discussed as a promising target for the treatment of severe COVID-19-associated diseases (Yap et al. 2020).

Hence, when DAMPs are emitted in a controlled fashion, they trigger innate immune pathways leading to successful host defense against those viral infections, up to full patient's recovery (compare Fig. 4). However, when emitted in an uncontrolled and excessive way, DAMPs have been shown to drive hyperinflammatory responses, clinically manifested as life-threatening acute respiratory distress syndrome (ARDS) and sepsis/SIRS (Nakahira et al. 2013; Tseng et al. 2014; Ma et al. 2018) [reviewed in Kang et al. (2015) and Denning et al. (2019)]. Together, according to current notions, life-threatening courses of viral infections depend on uncontrolled and excessive emission of DAMPs. And one may hypothesize that superimposition of environmental factors (e.g., air PM, pollutants) acting as exogenous DAMPs with virus-induced endogenous DAMPs may promote a fatal course of COVID-19. In fact, this model may serve as a blueprint for other non-viral conditions proposed to (1) contribute to the development of SARS case fatality, and (2) to be associated with emission of DAMPs such as aging (Zhou et al. 2020; Huang et al. 2015; Feldman et al. 2015), smoking (Vardavas and Nikitara 2020; Pouwels et al. 2016; Pouwels et al. 2016; Berlin et al. 2020), and diabetes (Kim and Lee 2020; Wang et al. 2020). Hence, one may insinuate: The DAMPs, emitted in excess as a result of two superimposing processes, and not the virus itself might be ultimately deadly.

In light of the exposome and AOP concepts, the following simplified scenario model can be sketched (Fig. 5): The catastrophic event of a SARS case fatality is promoted by superimposition of noninfectious environmental (e.g., air pollutants) and infectious (i.e., Coronaviruses as part of the "infectome") factors. The process starts with the sequelae of events (MIE $\rightarrow \mathrm{KE}_{4 \rightarrow n}$ ) as described for the controlled pathway (Fig. 4). However, instead of proceeding to homeostatic events, dysregulated excessive intrapulmonary emission of DAMPs, released from environmental noninfectious/ infectious factor-induced RCD, drive pathways, which now provoke "pathogenic" key events (KEs). In this example, the triggering "aberrant" event is the fifth KE that refers to pulmonary hyperinflammation, as characterized by DAMP (in excess)-activated, PRM-bearing inflammatory cells of the innate immune system (e.g., monocytes, macrophages, and neutrophils), executing various hyperinflammatory effector responses [see Land (2018) Chpt. 21, p. 473, Chpt. 22, p. 475, Chpt. 23, p. 591]. These events denoted as $\mathrm{KE}_{5 \rightarrow n}$, result in an AO in terms of ARDS. In this model, as KERs, massive/excessive secretion by DAMP $\leftrightarrow$ PRMactivated cells of proinflammatory mediator substances (cytokines, chemokines, adhesion molecules, others) is chosen $\left(\mathrm{KER}_{5 \rightarrow n}\right)$.

The sixth KE refers to systemic spreading of DAMPs in excess via the circulation, followed by DAMP $\leftrightarrow$ PRMmediated activation of innate immune cells, mediating hyperinflammation in various other organs $\left(\mathrm{KE}_{6 \rightarrow n}\right)$. As KERs, systemic secretion by DAMP $\leftrightarrow$ PRM-activated cells of proinflammatory mediator substances is chosen again $\left(\mathrm{KER}_{6 \rightarrow n}\right)$. These processes may proceed to SIRS/sepsis, associated with MODS, representing another AO. As KERs, 
secondary DAMP-promoted necroinflammatory pathways are chosen to act in this model $\left(\mathrm{KER}_{n}\right)$.

Finally, given the SARS CoV-2 outbreak considered a global pandemic, ARDS and SIRS/sepsis as typical AOs may be translated from an individual level to the population level (Fig. 5).

The Model of Environmental Factor-Induced Immune Diseases (Autoimmune, Allergic Diseases) In this model of immune disorders, the triggering "aberrant" event is $\mathrm{KE}_{4}$ that reflects migration of activated antigen (allergen/alteredself antigen) - presenting DCs from the peripheral inflammatory tissue to secondary lymphoid organs such as lymph nodes and spleen (Fig. 6). Thus, the fifth KE refers to a MAMP and/or DAMP-promoted, DC-triggered, antigendriven, specific adaptive $\mathrm{T}$ cell-/B cell-mediated immune response, characterized by proliferation, differentiation, and polarization of $\mathrm{T}$ cells as well as differentiation of $\mathrm{B}$ cells into plasma cells including isotope switching [compare Land (2018), Chpt. 31, p. 723 and Chpt. 32, 749]. The event sequence of $\mathrm{KE}_{5 \rightarrow \mathrm{n}}$ indicates the various antigenspecific adaptive responses, for example, allergen-specific or altered-self antigen (autoantigen)-specific responses that originate from the secondary lymphoid tissue/organs. Also, the various cellular T helper (Th) cell/humoral B cellmediated immune responses can be regarded as different KEs $\left(\mathrm{KE}_{5 \rightarrow n}\right)$. As $\mathrm{KER}_{5 \rightarrow n}$, DAMP-activated, PRM-bearing antigen-presenting DCs are elected, which lead to $\mathrm{T}$ cell priming.

The AO refers to manifest clinical symptoms of MAMP and/or DAMP-promoted, DC-triggered, antigen-driven human immune disorders such as autoimmune or allergic diseases. The various effector functions of activated $\mathrm{T}$ and B cells can be regarded as $\mathrm{KER}_{\mathrm{n}}$, mediated, for example, by cytotoxic T lymphocytes (CTLs) and cytotoxic (auto) antibodies.

The Model of Environmental Factor-Promoted Development of Organ Fibrosis A tentative translation of the exposome and AOP concepts into stress/injury $\rightarrow$ DAMP-promoted dysregulated pathways leading to fibrotic diseases again starts with the sequelae of events $\left(\mathrm{MIE} \rightarrow \mathrm{KE}_{4 \rightarrow n}\right)$ as described for the controlled pathway (Fig. 4). However, instead of proceeding to homeostatic events, the DAMPinduced pathways now provoke "pathogenic" KEs key events resulting in an $\mathrm{AO}$ in terms of organ fibrosis (Fig. 7). In this example, the triggering "aberrant" event is the fifth $\mathrm{KE}$ that refers to (uncontrolled) chronic inflammation as characterized by the failure of an inflammation-resolution response (="nonresolving inflammation"). The process may depend on poor generation of SAMPs in parallel to persistent or acutely repetitive emission of DAMPs, which proceed to activate inflammatory PRM-bearing monocytes, macrophages, and neutrophils, that is, events denoted as $\mathrm{KE}_{5 \rightarrow n}$ [compare Land (2018), Chpt. 22. p. 475]. As KERs, secretion by DAMP $\leftrightarrow$ PRM-activated macrophages and neutrophils of TGF- $\beta$ and other profibrotic cytokines is cho$\operatorname{sen}\left(\mathrm{KER}_{5 \rightarrow n}\right)$.

The sixth KE refers to DAMP $\leftrightarrow$ PRM-mediated activation of innate immune cells, in particular, macrophages that now produce TGF- $\beta$ and other profibrotic cytokines, thereby directly promoting fibroblast proliferation and differentiation, indicating the beginning of fibrogenesis. As KERs, secretion by macrophages and neutrophils of TGF- $\beta$, as well as other profibrotic cytokines are chosen $\left(\mathrm{KER}_{6 \rightarrow n}\right)$ [see Land (2018), Chpt. 36, p. 845].

The AO refers to the final stage of DAMP $\leftrightarrow$ PRMmediated tissue remodeling resulting in complete organ fibrosis associated with typical organ-specific clinical symptoms. As KERs, secretion by DAMP-activated fibrogenic cells (such as fibroblasts or hepatic stellate cells) of ECM compounds such as collagen are chosen to act $\left(\mathrm{KER}_{n}\right)$ [see Land (2018), Chpt. 36, p. 845].

Environmental Factor-Promoted Carcinogenesis: Sketching a Model for Cancer A tentative translation of the exposome and AOP concepts into stress/injury $\rightarrow$ DAMPpromoted dysregulated pathways eventually leading to carcinogenesis again starts with the sequelae of events $\left(\mathrm{MIE} \rightarrow \mathrm{KE}_{4 \rightarrow n}\right)$ as described for the controlled pathway (Fig. 4). However, instead of proceeding to homeostatic events, the DAMP-induced pathways now provoke "pathogenic" KEs key events resulting in an AO in terms of cancer (Fig. 8). In this example, the triggering "aberrant" event is again the fifth $\mathrm{KE}$ that refers to (uncontrolled) chronic inflammation as characterized by the failure of an inflammation-resolution response (= "nonresolving inflammation").

Indeed, epidemiological studies have shown that chronic inflammation can lead to a significant increase in the incidence of cancer, particularly tumors of epithelial origin. On the other hand, chronic inflammation is known to be caused and sustained by infectious/sterile injurious processes; in particular, when associated with regulated necrosis (RN), a phenomenon called necroinflammation (Sarhan et al. 2018). Typical examples are chronic Helicobacter pylori and Hepatitis $C$ Virus infections and exposure to toxic agents such as cigarette smoke, asbestos, and metal [for reviews, see Balkwill and Mantovani (2001), Hecht (2002), Grivennikov et al. (2010), Ben-Neriah and Karin (2011), Wang et al. (2016) and Zhang and $\mathrm{Xu}$ (2017)].

The exact mechanisms underlying chronic inflammatory microenvironment-promoted carcinogenesis are not fully understood. Nevertheless, it is a complex molecular process. 
Most convincing evidence, however, stems from studies suggesting that chronic infective/sterile injurious processes are not only associated with chronic inflammation but also can cause genetic changes (e.g., ROS-induced mutagenesis) and epigenetic alterations in normal but especially dormant malignant cells (Wang et al. 2016; Zhang and Xu 2017; Feinberg et al. 2016; Maiuri and O'Hagan 2016; Hattori and Ushijima 2016; Manjili 2017). Interestingly, as competently argued by Feinberg et al. (2016), there are certain cancer genes, the so-called epigenetic modulators, which serve as the mechanism by which environmental infectious or sterile stress/injury (e.g., oxidative stress/injury) transform tissues towards a neoplastic propensity and/or increase the likelihood that an accidental key mutation may lead to carcinogenesis. The authors suggest "that changes in the structure of chromatin are induced very early in the cancer process by epigenetic modulators and even in the non-mutated normal tissues from which tumors arise. Epigenetic modulator genes include many genes with prominent roles in conventional oncogenic signaling; these are increasingly appreciated to influence the epigenome as part of their function" (Feinberg et al. 2016).

Since DAMPs are known to promote and sustain chronic inflammation on the one hand, and since first evidence suggests that these molecules promote epigenetic modifications (Crisan et al. 2016), on the other hand, it is plausible to assume that they are also involved in chronic stress/injuryinduced carcinogenesis. Indeed, one may imagine that a series of genetic and epigenetic alterations associated with subsequent dysregulation of transcriptional, translational, and metabolic events may occur based on aberrant signaling pathways induced by chronically emitted constitutive and inducible DAMPs [for constitutive and inducible DAMPs, see Land (2018), Chpts. 11-16, pp. 191-370]. Such a suggestion is supported by evidence reviewed by Hernandez et al. (2016) arguing that "not only is there a strong correlation between DAMP expression and carcinogenesis in multiple tumors, but a plethora of DAMPs have been implicated in promoting inflammation and tumor development both in early stages of carcinogenesis as well as in established tumors."

\section{Résumé}

Certainly, this scenario model approach to combine the exposome and AOP concepts with the DAMPs narrative is incomplete and not perfect but should represent a first step to integrate these unique molecules and their pathologymediating functions into these two prominent environmental health research paradigms. It is hoped that this endeavor may contribute to the broad, multidisciplinary, fast-growing, and extremely active areas of environmental science and environmental (bio)technology. The final future aim of this approach certainly is to develop innovative DAMP-based in vitro tests for cumulative risk assessment, thereby allowing avoidance of the use of costly and (potentially) ethicallyflawed animal experiments. Since-as stressed by Smith et al. (2015)_-"the goal of cumulative risk assessment is to analyze, characterize, and quantify the combined risks to health or the environment from exposures to multiple agents or stressors," ... the junction of DAMPs with exposomics would be ... "perfectly poised to advance this important area of environmental health science."

\section{Conclusion and Outlook}

The scenario models described here suggest that the "DAMPome" may not only be the third concept in environmental science but may even unify the two other concepts. Even more so, the model gives rise to an interesting tautological discussion on the question: "Is research on (exogenous/endogenous) DAMP-induced innate immune pathways and DAMP — shaped adaptive immune processes just a particular part of environmental health research or even the same topic." Such an idea of tautology cannot be plausibly and rigorously excluded at present. Future interdisciplinary research has to be initiated and performed between the protagonists of all three research disciplines. Common interdisciplinary exchange of knowledge, as well as the organization of common scientific meetings, should be helpful in finally answering this question.

Author contributions WGL is the sole author.

Funding Not applicable.

\section{Compliance with Ethical Standards}

Conflict of interest The author declares that he has no competing interests.

\section{References}

Ankley GT, Bennett RS, Erickson RJ, Hoff DJ, Hornung MW, Johnson RD et al (2010) Adverse outcome pathways: a conceptual framework to support ecotoxicology research and risk assessment. Environ Toxicol Chem [Internet] 29:730-741. https://doi. org/10.1002/etc. 34

Aopwiki (2020) This wiki is hosted by the Society for the Advancement of Adverse Outcome Pathways (SAAOP) and serves as one component of a larger OECD-sponsored AOP Knowledgebase (AOP-KB) effort. [Internet]. https://aopwiki.org/; https://aopwi ki.org/info_pages/1/info_linked_pages/8

Arias-Reyes C, Zubieta-DeUrioste N, Poma-Machicao L, Aliaga-Raduan F, Carvajal-Rodriguez F, Dutschmann M et al (2020) Does 
the pathogenesis of SARS-CoV-2 virus decrease at high-altitude? Respir Physiol Neurobiol [Internet] 277:103443

Balkwill F, Mantovani A (2001) Inflammation and cancer: back to virchow. Lancet (Lond Engl) [Internet] 357:539-545

Becker RA, Ankley GT, Edwards SW, Kennedy SW, Linkov I, Meek B et al (2015) Increasing scientific confidence in adverse outcome pathways: application of tailored bradford-hill considerations for evaluating weight of evidence. Regul Toxicol Pharmacol [Internet] 72:514-537

Ben-Neriah Y, Karin M (2011) Inflammation meets cancer, with $\mathrm{NF}-\kappa \mathrm{B}$ as the matchmaker. Nat Immunol [Internet] 12:715-723. https://doi.org/10.1038/ni.2060

Berlin I, Thomas D, Le Faou A-L, Cornuz J (2020) COVID-19 and smoking. Nicotine Tob Res [Internet]. https://doi.org/10.1093/ ntr/ntaa059/5815378

Bogdanos DP, Sakkas LI (2017) From microbiome to infectome in autoimmunity [Internet]. Curr Opin Rheumatol 29:369-373. http://www.ncbi.nlm.nih.gov/pubmed/28394824

Bogdanos DP, Smyk DS, Invernizzi P, Rigopoulou EI, Blank M, Pouria $S$ et al (2013a) Infectome: a platform to trace infectious triggers of autoimmunity. Autoimmun Rev [Internet] 12:726-740. http:// www.ncbi.nlm.nih.gov/pubmed/23266520

Bogdanos DP, Smyk DS, Invernizzi P, Rigopoulou EI, Blank M, Sakkas L et al (2013b) Tracing environmental markers of autoimmunity: Introducing the infectome. Immunol Res [Internet] 56:220-240. http://www.ncbi.nlm.nih.gov/pubmed/23592050

Burden N, Sewell F, Andersen ME, Boobis A, Chipman JK, Cronin MTD et al (2015) Adverse outcome pathways can drive non-animal approaches for safety assessment. J Appl Toxicol [Internet] 35:971-975. https://doi.org/10.1002/jat.3165

Cai J, Zhao C, Du Y, Huang Y, Zhao Q (2019) Amentoflavone ameliorates cold stress-induced inflammation in lung by suppression of C3/BCR/NF- $\mathrm{BB}$ pathways. BMC Immunol [Internet] 20:49. http://www.ncbi.nlm.nih.gov/pubmed/31888465

Chen X, Guo X, Ge Q, Zhao Y, Mu H, Zhang J (2019a) ER stress activates the NLRP3 inflammasome: a novel mechanism of atherosclerosis. Oxid Med Cell Longev [Internet] 2019:1-18. https ://www.hindawi.com/journals/omcl/2019/3462530/

Chen I-Y, Moriyama M, Chang M-F, Ichinohe T (2019b) Severe acute respiratory syndrome coronavirus viroporin $3 \mathrm{a}$ activates the NLRP3 inflammasome. Front Microbiol [Internet] 10:50. http:// www.ncbi.nlm.nih.gov/pubmed/30761102

Colomina M-T, Sánchez-Santed F, Conejo NM, Collado P, Salvador A, Gallo M et al (2018) The Psychoexposome: a holistic perspective beyond health and disease. Psicothema [Internet] 30:5-7. http:// www.ncbi.nlm.nih.gov/pubmed/29363463

Conticini E, Frediani B, Caro D (2020) Can atmospheric pollution be considered a co-factor in extremely high level of SARS-CoV-2 lethality in Northern Italy? Environ Pollut [Internet] 261:114465

Crisan TO, Netea MG, Joosten LAB (2016) Innate immune memory: implications for host responses to damage-associated molecular patterns [Internet]. Eur J Immunol. https://doi.org/10.1002/ eji.201545497

Croft DP, Zhang W, Lin S, Thurston SW, Hopke PK, Masiol M et al (2019) The association between respiratory infection and air pollution in the setting of air quality policy and economic change. Ann Am Thorac Soc [Internet] 16:321-30. http://www.ncbi.nlm. nih.gov/pubmed/30398895

Cui Y, Zhang Z-F, Froines J, Zhao J, Wang H, Yu S-Z et al (2003) Air pollution and case fatality of SARS in the People's Republic of China: an ecologic study. Environ Heal [Internet] 2:15. https:// doi.org/10.1186/1476-069X-2-15

Dai M-Y, Chen F-F, Wang Y, Wang M-Z, Lv Y-X, Liu R-Y (2020a) Particulate matters induce acute exacerbation of allergic airway inflammation via the TLR2/NF- $\mathrm{BB} / \mathrm{NLRP} 3$ signaling pathway. Toxicol Lett [Internet] 321:146-154
Dai M-Y, Chen F-F, Wang Y, Wang M-Z, Lv Y-X, Liu R-Y (2020) Particulate matters induce acute exacerbation of allergic airway inflammation via the TLR2/NF- $\mathrm{KB} / \mathrm{NLRP} 3$ signaling pathway. Toxicol Lett [Internet] 321:146-154. http://www.ncbi.nlm.nih. gov/pubmed/31836503

Denning N-L, Aziz M, Gurien SD, Wang P (2019) DAMPs and NETs in sepsis. Front Immunol [Internet] 10:2536. http://www.ncbi. nlm.nih.gov/pubmed/31736963

Edwards SW, Tan Y-M, Villeneuve DL, Meek ME, McQueen CA (2016) Adverse outcome pathways-organizing toxicological information to improve decision making. J Pharmacol Exp Ther [Internet] 356:170-181. https://doi.org/10.1124/jpet.115.228239

Englert JA, Bobba C, Baron RM (2019) Integrating molecular pathogenesis and clinical translation in sepsis-induced acute respiratory distress syndrome. JCI Insight 4:e124061

Eppensteiner J, Kwun J, Scheuermann U, Barbas A, Limkakeng AT, Kuchibhatla M et al (2019) Damage- and pathogen-associated molecular patterns play differential roles in late mortality after critical illness. JCI Insight 4:e127925

Escher BI, Hackermüller J, Polte T, Scholz S, Aigner A, Altenburger $\mathrm{R}$ et al (2017) From the exposome to mechanistic understanding of chemical-induced adverse effects. Environ Int [Internet] 99:97-106. http://www.ncbi.nlm.nih.gov/pubmed/27939949

Feinberg AP, Koldobskiy MA, Göndör A (2016) Epigenetic modulators, modifiers and mediators in cancer aetiology and progression. Nat Rev Genet [Internet] 17:284-299. http://www.natur e.com/articles/nrg.2016.13

Feldman N, Rotter-Maskowitz A, Okun E (2015) DAMPs as mediators of sterile inflammation in aging-related pathologies. Ageing Res Rev [Internet] 24:29-39. http://www.ncbi.nlm.nih.gov/ pubmed/25641058

Fleshner M, Campisi J, Amiri L, Diamond DM (2004) Cat exposure induces both intra- and extracellular Hsp72: the role of adrenal hormones. Psychoneuroendocrinology [Internet] 29:1142-1152

Gallo PM, Gallucci S (2013) The dendritic cell response to classic, emerging, and homeostatic danger signals. Implications for autoimmunity. Front Immunol [Internet]. 4:138. http://www.ncbi. nlm.nih.gov/pubmed/23772226

Go Y-M, Jones DP (2017) Redox theory of aging: implications for health and disease. Clin Sci [Internet] 131:1669-1688. http:// www.ncbi.nlm.nih.gov/pubmed/28667066

Goldfeder RL, Wall DP, Khoury MJ, Ioannidis JPA, Ashley EA (2017) Human genome sequencing at the population scale: a primer on high-throughput DNA sequencing and analysis. Am J Epidemiol [Internet] 186:1000-1009. http://www.ncbi.nlm.nih.gov/pubme d/29040395

Goodwin S, McPherson JD, McCombie WR (2016) Coming of age: ten years of next-generation sequencing technologies. Nat Rev Genet [Internet] 17:333-351. http://www.nature.com/articles/ nrg.2016.49

Green ED, Guyer MS (2011) National Human Genome Research Institute. Charting a course for genomic medicine from base pairs to bedside. Nature [Internet] 470:204-213. http://www.nature.com/ articles/nature09764

Grivennikov SI, Greten FR, Karin M (2010) Immunity, inflammation, and cancer. Cell [Internet] 140:883-899. http://www.ncbi.nlm. nih.gov/pubmed/20303878

Hattori N, Ushijima T (2016) Epigenetic impact of infection on carcinogenesis: mechanisms and applications. Genome Med [Internet] 8:10. http://www.ncbi.nlm.nih.gov/pubmed/26823082

Hecht SS (2002) Cigarette smoking and lung cancer: chemical mechanisms and approaches to prevention. Lancet Oncol [Internet] 3:461-469. http://www.ncbi.nlm.nih.gov/pubmed/12147432

Hedbrant A, Andersson L, Bryngelsson I-L, Eklund D, Westberg H, Särndahl E et al (2020) Quartz dust exposure affects NLRP3 inflammasome activation and plasma levels of IL-18 and IL-1Ra 
in iron foundry workers. Mediators Inflamm [Internet] 2020:110. https://www.hindawi.com/journals/mi/2020/8490908/

Heil M, Land WG (2014) Danger signals_-damaged-self recognition across the tree of life. Front Plant Sci [Internet]. 5:578. http:// www.ncbi.nlm.nih.gov/pubmed/25400647

Heo MJ, Kim TH, You JS, Blaya D, Sancho-Bru P, Kim SG (2019) Alcohol dysregulates miR-148a in hepatocytes through FoxO1, facilitating pyroptosis via TXNIP overexpression. Gut [Internet] 68:708-720. http://www.ncbi.nlm.nih.gov/pubmed/29475852

Hernandez C, Huebener P, Schwabe RF (2016) Damage-associated molecular patterns in cancer: a double-edged sword. Oncogene [Internet] 35:5931-5941. http://www.ncbi.nlm.nih.gov/pubme $\mathrm{d} / 27086930$

Horvat T, Landesmann B, Lostia A, Vinken M, Munn S, Whelan M (2017) Adverse outcome pathway development from protein alkylation to liver fibrosis. Arch Toxicol [Internet] 91:15231543. https://doi.org/10.1007/s00204-016-1814-8

Huang J, Xie Y, Sun X, Zeh HJ, Kang R, Lotze MT et al (2015) DAMPs, ageing, and cancer: the "DAMP Hypothesis". Ageing Res Rev [Internet] 24:3-16. http://www.ncbi.nlm.nih.gov/pubme $\mathrm{d} / 25446804$

Hudson L, Rashdan E, Bonn CA, Chavan B, Rawlings D, Birch-Machin MA (2020) Individual and combined effects of the infrared, visible, and ultraviolet light components of solar radiation on damage biomarkers in human skin cells. FASEB J [Internet] 34:3874-3883. https://doi.org/10.1096/fj.201902351RR

Johansen Taber KA, Dickinson BD, Wilson M (2014) The promise and challenges of next-generation genome sequencing for clinical care. JAMA Intern Med [Internet] 174:275. http://www.ncbi.nlm. nih.gov/pubmed/24217348

Johnson CH, Athersuch TJ, Collman GW, Dhungana S, Grant DF, Jones DP et al (2017) Yale school of public health symposium on lifetime exposures and human health: the exposome; summary and future reflections. Hum Genom [Internet] 11:32. http://www. ncbi.nlm.nih.gov/pubmed/29221465

Kaczmarek A, Vandenabeele P, Krysko DV (2013) Necroptosis: the release of damage-associated molecular patterns and its physiological relevance. Immunity [Internet] 38:209-23. http://www. ncbi.nlm.nih.gov/pubmed/23438821

Kan HD, Chen BH, Fu CW, Yu SZ, Mu L (2005) Relationship between ambient air pollution and daily mortality of SARS in Beijing. [Internet]. Biomed Environ Sci 18:1-4. https://www.ncbi. nlm.nih.gov/pubmed/?term=Relationship+between+ambie nt+air+pollution+and+daily+mortality+of+SARS+in+Beijing

Kang J-W, Kim S-J, Cho H-I, Lee S-M (2015) DAMPs activating innate immune responses in sepsis. Ageing Res Rev [Internet] 24:54-65

Kim TK, Lee M-S (2020) Innate immune receptors in type 1 diabetes: the relationship to cell death-associated inflammation. Biochem Soc Trans [Internet] 48:1213-1225. https://portlandpress.com/ biochemsoctrans/article/48/3/1213/225192/Innate-immune-recep tors-in-type-1-diabetes-the

Land W (2003) Allograft injury mediated by reactive oxygen species: from conserved proteins of drosophila to acute and chronic rejection of human transplants Part III: interaction of (oxidative) stress-induced heat shock proteins with Toll-like receptorbearing cells. Transplant Rev [Internet] 17:67-86

Land WG (2018) Damage-associated molecular patterns in human diseases; vol. 1: injury-induced innate immune responses [Internet]. Springer International Publishing AG, New York. https://www. springer.com/de/book/9783319786544

Land WG, Messmer K (2012) The danger theory in view of the injury hypothesis: 20 years later. Front Immunol [Internet] 3:349. https ://doi.org/10.3389/fimmu.2012.00349/abstract

Land WG, Messmer K (2013) The innate immune system: its rediscovery before toll was described [Internet]. In: Innate immunity: resistance and disease-promoting principles. Karger Publishers, Berlin, p 24-28. https://doi.org/10.1159/000346531

Land W, Schneeberger H, Schleibner S, Illner WD, Abendroth D, Rutili G et al (1994) The beneficial effect of human recombinant superoxide dismutase on acute and chronic rejection events in recipients of cadaveric renal transplants. Transplantation [Internet] 57:211-217. http://www.ncbi.nlm.nih.gov/pubmed/8310510

Land WG, Agostinis P, Gasser S, Garg AD, Linkermann A (2016) Transplantation and damage associated molecular patterns (DAMPs). Am J Transplant [Internet] 16:3338-3361. http:// www.ncbi.nlm.nih.gov/pubmed/27421829

Leist M, Ghallab A, Graepel R, Marchan R, Hassan R, Bennekou SH et al (2017) Adverse outcome pathways: opportunities, limitations and open questions. Arch Toxicol [Internet] 91:3477-3505. https://doi.org/10.1007/s00204-017-2045-3

Lerner AG, Upton J-P, Praveen PVK, Ghosh R, Nakagawa Y, Igbaria $A$ et al (2012) IRE1 $\alpha$ induces thioredoxin-interacting protein to activate the NLRP3 inflammasome and promote programmed cell death under irremediable ER stress. Cell Metab [Internet] 16:250-264. http://www.ncbi.nlm.nih.gov/pubmed/22883233

Liaw PC, Ito T, Iba T, Thachil J, Zeerleder S (2016) DAMP and DIC: the role of extracellular DNA and DNA-binding proteins in the pathogenesis of DIC [Internet]. Blood Rev 30:257-261. https:// doi.org/10.1016/j.blre.2015.12.004

Lioy PJ, Smith KR (2013) A discussion of exposure science in the 21 st century: a vision and a strategy. Environ Health Perspect [Internet] 121:405-419. http://www.ncbi.nlm.nih.gov/pubme $\mathrm{d} / 23380895$

Liu R, Xu F, Bi S, Zhao X, Jia B, Cen Y (2019) Mitochondrial DNAinduced inflammatory responses and lung injury in thermal injury murine model: protective effect of cyclosporine-A. J Burn Care Res [Internet] 40:355-360. http://www.ncbi.nlm. nih.gov/pubmed/30926991

Ma KC, Schenck EJ, Siempos II, Cloonan SM, Finkelsztein EJ, Pabon MA et al (2018) Circulating RIPK3 levels are associated with mortality and organ failure during critical illness. JCI Insight 3:e99692

Maiuri AR, O'Hagan HM (2016) Interplay between inflammation and epigenetic changes in cancer. Prog Mol Biol Transl Sci 144:69-117

Manjili MH (2017) Tumor dormancy and relapse: from a natural byproduct of evolution to a disease state. Cancer Res [Internet] 77:2564-2569. http://www.ncbi.nlm.nih.gov/pubmed/28507050

Manrai AK, Cui Y, Bushel PR, Hall M, Karakitsios S, Mattingly CJ et al (2017) Informatics and data analytics to support exposomebased discovery for public health. Annu Rev Public Health [Internet] 38:279-294. https://doi.org/10.1146/annurev-publh ealth-082516-012737

Matzinger P (1994) Tolerance, danger, and the extended family. Annu Rev Immunol [Internet] 12:991-1045. http://www.ncbi.nlm.nih. gov/pubmed/8011301

Matzinger P (2012) The evolution of the danger theory. Interview by Lauren constable, commissioning editor. Expert Rev Clin Immunol [Internet] 8:311-317. http://www.ncbi.nlm.nih.gov/pubme $\mathrm{d} / 22607177$

Miller GW, Jones DP (2014) The nature of nurture: refining the definition of the exposome. Toxicol Sci [Internet]. 137:1-2. https:// academic.oup.com/toxsci/article/1647257/The

Moloudizargari M, Moradkhani F, Asghari N, Fallah M, Asghari MH, Moghadamnia AA et al (2019) NLRP inflammasome as a key role player in the pathogenesis of environmental toxicants [Internet]. Life Sci 231:116585. http://www.ncbi.nlm.nih.gov/pubme d/31226415

Moon Y (2016) Microbiome-linked crosstalk in the gastrointestinal exposome towards host health and disease. Pediatr Gastroenterol 
Hepatol Nutr [Internet] 19:221. http://www.ncbi.nlm.nih.gov/ pubmed/28090466

Moriyama M, Hugentobler WJ, Iwasaki A (2020) Seasonality of respiratory viral infections. Annu Rev Virol [Internet] http://www. ncbi.nlm.nih.gov/pubmed/32196426

Nagata S, Tanaka M (2017) Programmed cell death and the immune system [Internet]. Nat Rev Immunol 17:333-40. http://www.ncbi. nlm.nih.gov/pubmed/28163302

Nailwal H, Chan FK-M (2019) Necroptosis in anti-viral inflammation. Cell Death Differ [Internet] 26:4-13. http://www.ncbi.nlm.nih. gov/pubmed/30050058

Nakahira K, Kyung S-Y, Rogers AJ, Gazourian L, Youn S, Massaro AF et al (2013) Circulating mitochondrial DNA in patients in the ICU as a marker of mortality: derivation and validation. PLoS Med [Internet] 10:e1001577. https://doi.org/10.1371/journ al.pmed.1001577

Nakamura J, Mutlu E, Sharma V, Collins L, Bodnar W, Yu R et al (2014) The endogenous exposome. DNA Repair (Amst) [Internet] 19:3-13. http://www.ncbi.nlm.nih.gov/pubmed/24767943

National Human Genome (2018) All About The Human Genome Project (HGP) - National Human Genome Research Institute (NHGRI) [Internet]. https://www.genome.gov/10001772/

National Research Council (NRC) (2011) Toward precision medicine [Internet]. National Academies Press, Washington, D.C. http:// www.nap.edu/catalog/13284

National Research Council (NRC) (2012) Exposure Science in the 21st Century [Internet]. National Academies Press, Washington, D.C. http://www.nap.edu/catalog/13507

Netea MG, van der Meer JWM (2017) Trained immunity: an ancient way of remembering. Cell Host Microbe [Internet] 21:297-300

Netea MG, Joosten LAB, Latz E, Mills KHG, Natoli G, Stunnenberg HG et al (2016) Trained immunity: a program of innate immune memory in health and disease. Science [Internet] 352:aaf1098 http://www.ncbi.nlm.nih.gov/pubmed/27102489

Newton K (2015) RIPK1 and RIPK3: critical regulators of inflammation and cell death. Trends Cell Biol [Internet] 25:347-353

OECD (2014) The Adverse Outcome Pathway for Skin Sensitisation Initiated by Covalent Binding to Proteins [Internet]. OECD Publishing, Paris http://www.oecd-ilibrary.org/environment/ guidance-document-on-the-reporting-of-defined-approaches -and-individual-information-sources-to-be-used-within-integ rated-approaches-to-testing-and-assessment-iata-for-skin-sensi tisation_9789264279285-en

OECD (2018) Users' handbook supplement to the guidance document for developing and assessing adverse outcome pathways. OECD iLibrary. https://www.oecd-ilibrary.org/environment/users-handb ook-supplement-to-the-guidance-document-for-developing-andassessing-adverse-outcome-pathways_5jlv1m9d1g32-en

Otani T, Noma H, Nishino J, Matsui S (2018) Re-assessment of multiple testing strategies for more efficient genome-wide association studies. Eur J Hum Genet [Internet] 26:1038-1048. http://www. nature.com/articles/s41431-018-0125-3

Patel CJ (2017) Analytic complexity and challenges in identifying mixtures of exposures associated with phenotypes in the exposome era. Curr Epidemiol Rep [Internet] 4:22-30. http://www.ncbi. nlm.nih.gov/pubmed/28251040

Patel MC, Shirey KA, Boukhvalova MS, Vogel SN, Blanco JCG (2018) Serum high-mobility-group box 1 as a biomarker and a therapeutic target during respiratory virus infections. MBio [Internet]. https://doi.org/10.1128/mBio.00246-18

Perkins EJ, Antczak P, Burgoon L, Falciani F, Garcia-Reyero N, Gutsell S et al (2015) Adverse outcome pathways for regulatory applications: examination of four case studies with different degrees of completeness and scientific confidence. Toxicol Sci [Internet] 148:14-25. https://doi.org/10.1093/toxsci/kfv181
Pouwels SD, Zijlstra GJ, van der Toorn M, Hesse L, Gras R, ten Hacken NHT et al (2016) Cigarette smoke-induced necroptosis and DAMP release trigger neutrophilic airway inflammation in mice. Am J Physiol Cell Mol Physiol [Internet] 310:L377-L386. http://www.ncbi.nlm.nih.gov/pubmed/26719146

Pouwels SD, Hesse L, Faiz A, Lubbers J, Bodha PK, ten Hacken NHT et al (2016) Susceptibility for cigarette smoke-induced DAMP release and DAMP-induced inflammation in COPD. Am J Physiol Cell Mol Physiol [Internet] 311:L881-L892. http://www.ncbi. nlm.nih.gov/pubmed/27612964

Qu G, Li X, Hu L, Jiang G (2020) An imperative need for research on the role of environmental factors in transmission of novel coronavirus (COVID-19). Environ Sci Technol [Internet] 54:37303732. https://doi.org/10.1021/acs.est.0c01102

Ramu S, Menzel M, Bjermer L, Andersson C, Akbarshahi H, Uller L (2018) Allergens produce serine proteases-dependent distinct release of metabolite DAMPs in human bronchial epithelial cells. Clin Exp Allergy [Internet] 48:156-66. http://www.ncbi.nlm.nih. gov/pubmed/29210131

Rappaport SM (2011) Implications of the exposome for exposure science. J Expo Sci Environ Epidemiol [Internet] 21:5-9. http:// www.nature.com/articles/jes201050

Rappaport SM (2018) Redefining environmental exposure for disease etiology. NPJ Syst Biol Appl [Internet] 4:30. http://www.ncbi. nlm.nih.gov/pubmed/30181901

Rappaport SM, Smith MT (2010) Epidemiology. Environment and disease risks. Science 330:460-461

Rappaport SM, Barupal DK, Wishart D, Vineis P, Scalbert A (2014) The blood exposome and its role in discovering causes of disease. Environ Health Perspect [Internet] 122:769-774. http:// ehp.niehs.nih.gov/1308015

Rattray NJW, Deziel NC, Wallach JD, Khan SA, Vasiliou V, Ioannidis JPA et al (2018) Beyond genomics: understanding exposotypes through metabolomics. Hum Genom [Internet] 12:4. http://www.ncbi.nlm.nih.gov/pubmed/29373992

Relja B, Land WG (2019) Damage-associated molecular patterns in trauma. Eur J Trauma Emerg Surg [Internet]. https://doi. org/10.1007/s00068-019-01235-w

Sano R, Reed JC (2013) ER stress-induced cell death mechanisms. Biochim Biophys Acta Mol Cell Res [Internet] 1833:34603470. http://www.ncbi.nlm.nih.gov/pubmed/23850759

Santos S, Maitre L, Warembourg C, Agier L, Richiardi L, Basagaña $\mathrm{X}$ et al (2020) Applying the exposome concept in birth cohort research: a review of statistical approaches. Eur J Epidemiol [Internet] 35:193-204. https://doi.org/10.1007/s10654-02000625-4

Sarhan M, Land WG, Tonnus W, Hugo CP, Linkermann A (2018) Origin and consequences of necroinflammation. Physiol Rev [Internet] 98:727-780. https://doi.org/10.1152/physrev.00041 .2016

Sasaki JC, Allemang A, Bryce SM, Custer L, Dearfield KL, Dietz $Y$ et al (2020) Application of the adverse outcome pathway framework to genotoxic modes of action. Environ Mol Mutagen [Internet] 61:114-134. https://doi.org/10.1002/em.22339

Seong S-Y, Matzinger P (2004) Hydrophobicity: an ancient damage-associated molecular pattern that initiates innate immune responses. Nat Rev Immunol [Internet] 4:469-78. http://www. ncbi.nlm.nih.gov/pubmed/15173835

Sharp A, McPherson P, Dawson T, Aoki C, Campbell K, Snyder S (1993) Differential immunohistochemical localization of inositol 1,4,5-trisphosphate- and ryanodine-sensitive $\mathrm{Ca}^{2+}$ release channels in rat brain. J Neurosci [Internet] 13:3051-3063. https ://doi.org/10.1523/JNEUROSCI.13-07-03051.1993

Siroux V, Agier L, Slama R (2016) The exposome concept: a challenge and a potential driver for environmental health research. 
Eur Respir Rev [Internet] 25:124-129. http://www.ncbi.nlm. nih.gov/pubmed/27246588

Smith MT, de la Rosa R, Daniels SI (2015) Using exposomics to assess cumulative risks and promote health. Environ Mol Mutagen [Internet] 56:715-723. https://doi.org/10.1002/ em. 21985

Spinu N, Cronin MTD, Enoch SJ, Madden JC, Worth AP (2020) Quantitative adverse outcome pathway (qAOP) models for toxicity prediction. Arch Toxicol [Internet] 94:1497-1510. https://doi. org/10.1007/s00204-020-02774-7

Sun Z, Thilakavathy K, Kumar SS, He G, Liu S V (2020) Potential factors influencing repeated SARS outbreaks in China. Int $\mathbf{J}$ Environ Res Public Health [Internet] 17:1633. https://www.mdpi. com/1660-4601/17/5/1633

Swanson KV, Deng M, Ting JPY (2019) The NLRP3 inflammasome: molecular activation and regulation to therapeutics. Nat Rev Immunol [Internet] 19:477-89. http://www.nature.com/articles/ s41577-019-0165-0

Theodoratou E, Timofeeva M, Li X, Meng X, Ioannidis JPA (2017) Nature, nurture, and cancer risks: genetic and nutritional contributions to cancer. Annu Rev Nutr [Internet] 37:293-320. https ://doi.org/10.1146/annurev-nutr-071715-051004

Toh C-H, Alhamdi Y, Abrams ST (2016) Current pathological and laboratory considerations in the diagnosis of disseminated intravascular coagulation. Ann Lab Med [Internet] 36:505. https:// doi.org/10.3343/alm.2016.36.6.505

Tolle LB, Standiford TJ (2013) Danger-associated molecular patterns (DAMPs) in acute lung injury. J Pathol [Internet] 229:145-156. http://www.ncbi.nlm.nih.gov/pubmed/23097158

Tseng C-C, Fang W-F, Leung S-Y, Chen H-C, Chang Y-C, Wang C-C et al (2014) Impact of serum biomarkers and clinical factors on intensive care unit mortality and 6-month outcome in relatively healthy patients with severe pneumonia and acute respiratory distress syndrome. Dis Mark [Internet] 2014:804654. http://www. ncbi.nlm.nih.gov/pubmed/24723739

Uppal K, Walker DI, Liu K, Li S, Go Y-M, Jones DP (2016) Computational metabolomics: a framework for the million metabolome. Chem Res Toxicol [Internet] 29:1956-75. http://www.ncbi.nlm. nih.gov/pubmed/27629808

US EPA (2019) Framework for cumulative risk assessment. https:// www.epa.gov/risk/framework-cumulative-risk-assessment

van Bilsen JHM, Sienkiewicz-Szłapka E, Lozano-Ojalvo D, Willemsen LEM, Antunes CM, Molina E et al (2017) Application of the adverse outcome pathway (AOP) concept to structure the available in vivo and in vitro mechanistic data for allergic sensitization to food proteins. Clin Transl Allergy [Internet]. 7:13. https ://doi.org/10.1186/s13601-017-0152-0

Vardavas C, Nikitara K (2020) COVID-19 and smoking: a systematic review of the evidence. Tob Induc Dis [Internet] 18:20. http:// www.journalssystem.com/tid/COVID-19-and-smoking-A-syste matic-review-of-the-evidence, 119324,0,2.html

Vermeulen R, Schymanski EL, Barabási A-L, Miller GW (2020) The exposome and health: where chemistry meets biology. Science (80-) 367:392-396. https://doi.org/10.1126/science.aay3164

Villeneuve DL, Crump D, Garcia-Reyero N, Hecker M, Hutchinson TH, LaLone CA et al (2014) Adverse outcome pathway (AOP) development I: strategies and principles. Toxicol Sci [Internet]. 142:312-320. https://doi.org/10.1093/toxsci/kfu199

Vineis P, Avendano-Pabon M, Barros H, Bartley M, Carmeli C, Carra L et al (2020) Special report: the biology of inequalities in health: the lifepath consortium. Front Public Heal [Internet] 8:118. https ://doi.org/10.3389/fpubh.2020.00118/full

Vinken M, Knapen D, Vergauwen L, Hengstler JG, Angrish M, Whelan M (2017) Adverse outcome pathways: a concise introduction for toxicologists. Arch Toxicol [Internet] 91:3697-3707. https://doi. org/10.1007/s00204-017-2020-z

Wang L, Wise JTF, Zhang Z, Shi X (2016) Progress and prospects of reactive oxygen species in metal carcinogenesis. Curr Pharmacol Rep [Internet] 2:178-186. http://www.ncbi.nlm.nih.gov/pubme $\mathrm{d} / 27617186$

Wang X, Antony V, Wang Y, Wu G, Liang G (2020) Pattern recognition receptor-mediated inflammation in diabetic vascular complications. Med Res Rev [Internet]. https://doi.org/10.1002/ med.21711

Wild CP (2005) Complementing the genome with an "exposome": the outstanding challenge of environmental exposure measurement in molecular epidemiology. Cancer Epidemiol Biomark Prev [Internet] 14:1847-1850. http://www.ncbi.nlm.nih.gov/pubme $\mathrm{d} / 16103423$

Wild CP (2012) The exposome: from concept to utility. Int J Epidemiol [Internet] 41:24-32. https://doi.org/10.1093/ije/dyr236

Willart MAM, Poulliot P, Lambrecht BN, Kool M (2013) PAMPs and DAMPs in allergy exacerbation models. Methods Mol Biol 1032:185-204

Willett WC (2002) Balancing life-style and genomics research for disease prevention. Science [Internet] 296:695-698. https://doi. org/10.1126/science.1071055

Wilson SH, Schwartz DA (2006) Disease-first: a new paradigm for environmental health science research. Environ Health Perspect [Internet] 114:A398. http://www.ncbi.nlm.nih.gov/pubme $\mathrm{d} / 16835037$

Wu X, Nethery RC, Sabath BM, Braun D, Dominici F (2020) Exposure to air pollution and Covid-19 mortality in the United States. medRxiv [Internet]. https://doi.org/10.1101/2020.04.05.20054 $502 \mathrm{v} 1$

Yang Y, Peng F, Wang R, Guan K, Jiang T, Xu G et al (2020) The deadly coronaviruses: the 2003 SARS pandemic and the 2020 novel coronavirus epidemic in China. J Autoimmun 109:102434

Yap JKY, Moriyama M, Iwasaki A (2020) Inflammasomes and pyroptosis as therapeutic targets for COVID-19. J Immunol [Internet] 205:307-312. https://doi.org/10.4049/jimmunol.2000513

Yatim N, Cullen S, Albert ML (2017) Dying cells actively regulate adaptive immune responses. Nat Rev Immunol [Internet] 17:262-275. http://www.nature.com/articles/nri.2017.9

Yue Y, Nabar NR, Shi C-S, Kamenyeva O, Xiao X, Hwang I-Y et al (2018) SARS-coronavirus open reading frame-3a drives multimodal necrotic cell death. Cell Death Dis [Internet] 9:904. http:// www.nature.com/articles/s41419-018-0917-y

Zhang H, Xu X (2017) Mutation-promoting molecular networks of uncontrolled inflammation. Tumor Biol [Internet]. 39:101042831770131. http://www.ncbi.nlm.nih.gov/pubme $\mathrm{d} / 28653899$

Zhou F, Yu T, Du R, Fan G, Liu Y, Liu Z et al (2020) Clinical course and risk factors for mortality of adult inpatients with COVID-19 in Wuhan, China: a retrospective cohort study. Lancet [Internet] 395:1054-1062

Zhu Y, Xie J, Huang F, Cao L (2020) Association between short-term exposure to air pollution and COVID-19 infection: evidence from China. Sci Total Environ [Internet] 727:138704 